\title{
INFINITELY MANY HYPERELLIPTIC CURVES WITH EXACTLY TWO RATIONAL POINTS
}

\author{
YOSHINOSUKE HIRAKAWA AND HIDEKI MATSUMURA
}

\begin{abstract}
In this paper, we construct some families of infinitely many hyperelliptic curves of genus 2 with exactly two rational points. In the proof, we first show that the Mordell-Weil ranks of these hyperelliptic curves are 0 and then determine the sets of rational points by using the Lutz-Nagell type theorem for hyperelliptic curves which was proven by Grant.
\end{abstract}

\section{Contents}

1. Main theorem

2. 2-descent

2.1. Case $(i, j)=(0,1)$

2.2. Case $(i, j)=(1,1)$

2.3. Case $(i, j)=(0,2), p \equiv 3(\bmod 8)$

2.4. Case $(i, j)=(0,2), p \equiv-3(\bmod 8)$

3. Application of the Lutz-Nagell type theorem for hyperelliptic curves

4. Concluding remarks

Appendix A.

References

\section{Main theorem}

In this paper, we determine the set of rational points of the following hyperelliptic curves.

Theorem 1.1. Let $p$ be a prime number, $i, j \in \mathbb{Z}$, and $C^{(p ; i, j)}$ be a hyperelliptic curve defined by

$$
y^{2}=x\left(x^{2}+2^{i} p^{j}\right)\left(x^{2}+2^{i+1} p^{j}\right) .
$$

Suppose that one of the following conditions holds.

(1) $p \equiv 3(\bmod 16)$ and $(i, j)=(0,1)$.

(2) $p \equiv 11(\bmod 16)$ and $(i, j)=(1,1)$.

(3) $p \equiv 3(\bmod 8)$ and $(i, j)=(0,2)$.

(4) $p \equiv-3(\bmod 8)$ and $(i, j)=(0,2)$.

Then, $C^{(p ; i, j)}(\mathbb{Q})=\{(0,0), \infty\}$.

Date: April 3, 2019.

2010 Mathematics Subject Classification. primary 14G05; secondary 11G30.

Key words and phrases. rational points, hyperelliptic curves, 2-descent, Lutz-Nagell theorem.

This research was supported in part by KAKENHI 26247004, 18H05233 as well as the JSPS Core-toCore program "Foundation of a Global Research Cooperative Center in Mathematics focused on Number Theory and Geometry" and the KiPAS program FY2014-2018 of the Faculty of Science and Technology at Keio University. Both authors were supported by the Research Grant of Keio Leading-edge Laboratory of Science \& Technology 2018-2019 (Grant Numbers 000036 and 000053). 
Here, note that, in each case, there exist infinitely many prime numbers satisfying the above congruent condition by Dirichlet's theorem on arithmetic progressions. A striking feature of Theorem 1.1 is that we can treat infinitely many prime numbers $p$ which are "2-adically near to each other" simultaneously.

Our proof of Theorem 1.1 is based on natural generalizations of the 2-descent argument and the Lutz-Nagell theorem (cf. [11], [5, Theorem 3]). Recall that, in the case of elliptic curves, the 2-descent argument makes it possible to bound the Mordell-Weil rank of an elliptic curve by means of the 2-Selmer group, and the Lutz-Nagell theorem makes it possible to determine the torsion points of an elliptic curve by means of the discriminant. For example, by applying them, we can prove that the only rational points on an elliptic curve defined by $y^{2}=x(x+p)(x-p)$ with a prime number $p \equiv 3 \bmod 8$ are $(x, y)=$ $(0,0),(p, 0),(-p, 0)$ and $\infty$, i.e., such a prime number $p$ is never a congruent number (cf. [6, D27] and the references therein). Note that the 2-descent argument allows us to treat prime numbers $p$ which are "2-adically near to each other" simultaneously.

In $\S 2$, we prove that the Mordell-Weil rank of the Jacobian variety $J^{(p ; i, j)}$ of $C^{(p ; i, j)}$ is 0 by the 2-descent argument [11]. 11 Therefore, it is sufficient to determine the set of rational points on $C^{(p ; i, j)}$ which map to torsion points of $J^{(p ; i, j)}$ via the Abel-Jacobi map associated with the point at infinity $\infty$.

In $\S 3$, we carry out this task by applying the Lutz-Nagell type theorem for hyperelliptic curves which was proven by Grant [5]. 2

Remark 1.2. (1) If $p$ is a prime number which does not satisfy the above congruence condition, then $C^{(p ; i, j)}$ may have additional rational points. For instance, $C^{(17 ; 0,1)}$ has additional rational points $(8, \pm 252)$.

(2) Let $i, j \in \mathbb{Z}_{\geq 0}$ and $C^{(i, j)}$ be a hyperelliptic curve over $\mathbb{Q}(t)$ defined by

$$
y^{2}=x\left(x^{2}+2^{i} t^{j}\right)\left(x^{2}+2^{i+1} t^{j}\right) .
$$

Then, $C^{(i, j)}(\mathbb{Q}(t))=\{(0,0), \infty\}$. For $(i, j)=(0,1),(1,1),(0,2)$, this is a consequence of Theorem 1.1. In fact, we can deduce it easily from the abc conjecture for polynomials (cf. [10, Theorem]) for general $(i, j)$ (Appendix A).

\section{2-descent}

Let $p$ be a (odd) prime number, $i, j \in \mathbb{Z}$, and $f(x)=x\left(x^{2}+2^{i} p^{j}\right)\left(x^{2}+2^{i+1} p^{j}\right)$. Let $C^{(p ; i, j)}$ be a hyperelliptic curve defined by $y^{2}=f(x)$ and $J^{(p ; i, j)}$ be its Jacobian variety. In this section, we prove the following theorem.

Theorem 2.1. Suppose that one of the following conditions holds.

(1) $p \equiv 3(\bmod 16)$ and $(i, j)=(0,1)$.

(2) $p \equiv 11(\bmod 16)$ and $(i, j)=(1,1)$.

(3) $p \equiv 3(\bmod 8)$ and $(i, j)=(0,2)$.

(4) $p \equiv-3(\bmod 8)$ and $(i, j)=(0,2)$.

Then, we have $\operatorname{rank}\left(J^{(p ; i, j)}(\mathbb{Q})\right)=0$.

We treat the above four cases separately but in a similar manner in the following four subsections respectively. Since $J^{(p ; i, j)}(\mathbb{Q}) / 2 J^{(p ; i, j)}(\mathbb{Q})$ can be embedded into the 2-Selmer group $\operatorname{Sel}^{(2)}\left(\mathbb{Q}, J^{(p ; i, j)}\right)$, in order to bound the Mordell-Weil rank from above, it is sufficient

\footnotetext{
${ }^{1}$ The primary advantage of our families is that the verification of this fact is relatively easy.

${ }^{2}$ Since $\# C^{(p ; i, j)}\left(\mathbb{F}_{q}\right) \geq 4$ for every $p, i, j$ and another prime number $q \neq 2, p$, the Chabauty-Coleman method (cf. 2, Corollary 4a], 7. Theorem 5.3 (b)]) is not sufficient.
} 
to calculate the dimension of the 2-Selmer group. By [11, p. 256], we have

$$
\begin{aligned}
\operatorname{dim} \operatorname{Sel}^{(2)}\left(\mathbb{Q}, J^{(p ; i, j)}\right)= & \operatorname{dim} \operatorname{val}^{-1}(G)+\operatorname{dim}\left(\operatorname{Im}\left(\delta_{2}\right) \times \operatorname{Im}\left(\delta_{p}\right)\right) \\
& -\operatorname{dim}\left(\left(\operatorname{Im}\left(\delta_{2}\right) \times \operatorname{Im}\left(\delta_{p}\right)\right)+\operatorname{res}_{S}\left(\operatorname{val}^{-1}(G)\right)\right) .
\end{aligned}
$$

In each case, we can prove that the right hand side equals 2. $\left.{ }^{3}\right]^{4}$ Here and after, we follow the notation in [11 as below.

Notation 2.2. We fix $p$, $i$, and $j$, so we abbreviate $C^{(p ; i, j)}$ to $C$ and $J^{(p ; i, j)}$ to $J$. Denote

- the $x$-coordinate of the point $P \in C(\mathbb{Q})$ by $x_{P}$,

- every divisor class in $J(\mathbb{Q})$ represented by a divisor $D$ simply by $D$,

- a fixed algebraic closure of $\mathbb{Q}$ by $\overline{\mathbb{Q}}$.

For every place $v$, we also use a similar notation and fix an embedding $\overline{\mathbb{Q}} \hookrightarrow \overline{\mathbb{Q}} v$. Define

- $L:=\mathbb{Q}[T] /(f(T)) \stackrel{\simeq}{\rightarrow} \prod_{i=1}^{3} L^{(i)} ; T \mapsto\left(T_{1} ; T_{2} ; T_{3}\right)$, where $L^{(1)}:=\mathbb{Q}\left[T_{1}\right] /\left(T_{1}\right)$, $L^{(2)}:=\mathbb{Q}\left[T_{2}\right] /\left(T_{2}^{2}+2^{i} p^{j}\right)$ and $L^{(3)}:=\mathbb{Q}\left[T_{3}\right] /\left(T_{3}^{2}+2^{i+1} p^{j}\right)$. We denote the trivial elements in $L^{\times}$and $L^{\times} / L^{\times 2}$ by $\mathbf{1}$.

For every place $v$, define

- $L_{v}:=\mathbb{Q}_{v}[T] /(f(T)) \stackrel{\sim}{\rightarrow} \prod_{i=1}^{m} L_{v}^{(i)} ; T \mapsto\left(T_{1} ; \cdots ; T_{m}\right)$, where $f(T)=f_{1}(T) \cdots f_{m}(T)$ is the irreducible decomposition in $\mathbb{Q}_{v}[T]$ and $L_{v}^{(i)}:=\mathbb{Q}_{v}\left[T_{i}\right] /\left(f_{i}\left(T_{i}\right)\right)$. We denote the trivial elements in $L_{v}^{\times}$and $L_{v}^{\times} / L_{v}^{\times 2}$ by $\mathbf{1}_{v}$.

- $\delta_{v}: J\left(\mathbb{Q}_{v}\right) \rightarrow L_{v}^{\times} / L_{v}^{\times 2} ; D=\sum_{i=1}^{n} m_{i} P_{i} \mapsto \prod_{i=1}^{n}\left(x_{P_{i}}-T\right)^{m_{i}}$, where $D$ is a divisor whose support is disjoint from the support of the divisor $\operatorname{div}(y)$

- $\operatorname{res}_{v}: L^{\times} / L^{\times 2} \rightarrow L_{v}^{\times} / L_{v}^{\times 2}$ as the map induced by $L \rightarrow L_{v} ; T \mapsto T$.

- $I_{v}(L):=\prod_{i=1}^{3}$ (the group of fractional ideals of $\left.L_{v}^{(i)}\right)$. We often denote

- each element of $I_{v}(L)$ and $I_{v}(L) / I_{v}(L)^{2}$ by $\left(\mathfrak{a}^{(1)} ; \ldots ; \mathfrak{a}^{(m)}\right)_{v}$.

- the trivial elements of $I_{v}(L)$ and $I_{v}(L) / I_{v}(L)^{2}$ by $\mathbf{1}_{v}$.

- $\operatorname{val}_{v}: L_{v}^{\times} \rightarrow I_{v}(L) ; a \mapsto(a)$. 5 We denote the induced map $L_{v}^{\times} / L_{v}^{\times 2} \rightarrow I_{v}(L) / I_{v}(L)^{2}$ simply by $\operatorname{val}_{v}$.

- $G_{v}:=\operatorname{val}_{v}\left(\operatorname{Im}\left(\delta_{v}\right)\right) \subset I_{v}(L) / I_{v}(L)^{2}$.

Finally, define

- $I(L):=\prod_{v} I_{v}(L) \simeq \prod_{i=1}^{3}$ (the group of fractional ideals of $\left.L^{(i)}\right)$. We identify $I_{2}(L) \times$ $I_{p}(L)$ with its image in $I(L)$.

- $\mathrm{Cl}(L):=\prod_{i=1}^{3} \mathrm{Cl}\left(L^{(i)}\right)$, where $\mathrm{Cl}\left(L^{(i)}\right)$ are the ideal class groups of $L^{(i)}$.

- $S:=\{2, p, \infty\}$.

- $\operatorname{res}_{S}:=\prod_{v \in S \backslash\{\infty\}} \operatorname{res}_{v}: L^{\times} / L^{\times 2} \rightarrow \prod_{v \in S \backslash\{\infty\}} L_{v}^{\times} / L_{v}^{\times 2}$.

- val $:=\prod_{v} \operatorname{val}_{v} \circ \operatorname{res}_{v}: L^{\times} \rightarrow I(L)$. We denote the induced map $L^{\times} / L^{\times 2} \rightarrow$ $I(L) / I(L)^{2}$ simply by val.

- $G:=\prod_{v \in S \backslash\{\infty\}} G_{v} \subset I(L) / I(L)^{2}$.

- $W:=\operatorname{Ker}\left(G \rightarrow \mathrm{Cl}(L) / \mathrm{Cl}(L)^{2}\right)$.

${ }^{3}$ In other cases, computation by MAGMA 1 suggests that $\operatorname{dim}_{\mathbb{F}_{2}} \operatorname{Sel}^{(2)}\left(\mathbb{Q}, J^{(p ; i, j)}\right)>2$.

${ }^{4}$ Note that $\operatorname{Im}\left(\delta_{\infty}\right)$ is trivial. Note also that $\operatorname{dim}_{\mathbb{F}_{2}} \operatorname{Im}\left(\delta_{2}\right)\left(\right.$ resp. $\left.\operatorname{dim}_{\mathbb{F}_{2}} \operatorname{Im}\left(\delta_{p}\right)\right)$ is independent of prime number $p$. For the detail, see Lemmas 2.5, 2.6, 2.15, 2.16, 2.22, 2.23, 2.29 and 2.30. More strongly, $C^{(p ; i, j)}$ and $C^{(q ; i, j)}$ are isomorphic over $\mathbb{Q}_{2}$ whenever $p \equiv q(\bmod 16)$. Moreover, $C^{(p ; 0,2)}$ and $C^{(q ; 0,2)}$ are isomorphic over $\mathbb{Q}_{2}$ whenever $p^{2} \equiv q^{2}(\bmod 16)$.

${ }^{5}$ Let $\mathfrak{q}_{v}^{(i, 1)}, \ldots, \mathfrak{q}_{v}^{\left(i, j_{i}\right)}$ be the prime ideals of $L^{(i)}$ above $v$, then

$$
\operatorname{val}_{v}\left(\alpha_{1}, \ldots, \alpha_{m}\right)=\left(\alpha_{1}^{v\left(\mathfrak{q}_{v}^{\left(1, j_{1}\right)}\right)}, \ldots, \alpha_{m}^{v\left(\mathfrak{q}_{v}^{\left(1, j_{1}\right)}\right)} ; \ldots ; \alpha_{1}^{v\left(\mathfrak{q}_{v}^{(m, 1)}\right)}, \ldots, \alpha_{m}^{v\left(\mathfrak{q}_{v}^{\left(m, j_{m}\right)}\right)}\right)
$$

which is well-defined up to the order of $\mathfrak{q}_{v}^{(i, 1)}, \ldots, \mathfrak{q}_{v}^{\left(i, j_{i}\right)}$ for each $i$. Here, we identify the prime number $v$ and the associated valuation map. 
2.1. Case $(i, j)=(0,1)$

Suppose that $p \equiv 3(\bmod 16)$. Then, we have the following irreducible decompositions:

$$
\left\{\begin{array}{l}
L_{2}=\mathbb{Q}_{2}\left[T_{1}\right] /\left(T_{1}\right) \times \mathbb{Q}_{2}\left[T_{2}\right] /\left(T_{2}^{2}+p\right) \times \mathbb{Q}_{2}\left[T_{3}\right] /\left(T_{3}^{2}+2 p\right), \\
L_{p}=\mathbb{Q}_{p}\left[T_{1}\right] /\left(T_{1}\right) \times \mathbb{Q}_{p}\left[T_{2}\right] /\left(T_{2}^{2}+p\right) \times \mathbb{Q}_{p}\left[T_{3}\right] /\left(T_{3}^{2}+2 p\right) .
\end{array}\right.
$$

Lemma 2.3. The following two elements form an $\mathbb{F}_{2}$-basis of $J(\mathbb{Q})[2]$ and $J\left(\mathbb{Q}_{v}\right)[2]$ for $v=2, p, \infty$ :

$$
(0,0)-\infty, \quad \sum_{\substack{P \in C(\overline{\mathbb{Q}}) \\ x_{P}^{2}+p=0}} P-2 \infty .
$$

In particular, we have the following table. ${ }^{6}$

\begin{tabular}{|l||l|l|}
\hline$v$ & $\operatorname{dim} J\left(\mathbb{Q}_{v}\right)[2]$ & $\operatorname{dim} \operatorname{Im}\left(\delta_{v}\right)$ \\
\hline \hline 2 & 2 & 4 \\
\hline$p$ & 2 & 2 \\
\hline$\infty$ & 2 & 0 \\
\hline
\end{tabular}

Proof. The first statement follows from [12, Lemma 5.2]. Note that neither $-p$ nor $-2 p$ is not square in $\mathbb{Q}_{v}$ for $v=2, p, \infty$. The second statement follows from the following formula (cf. [4, p. 451, proof of Lemma 3]).

$$
\operatorname{dim}_{\mathbb{F}_{2}} \operatorname{Im}\left(\delta_{v}\right)=\operatorname{dim}_{\mathbb{F}_{2}} J\left(\mathbb{Q}_{v}\right)[2] \begin{cases}+0 & (v \neq 2, \infty), \\ +2 & (v=2), \\ -2 & (v=\infty) .\end{cases}
$$

In the calculation of $\operatorname{Im}\left(\delta_{v}\right)$, we use the following formula.

Lemma 2.4 ([9, Lemma 2.2]). Let $C: y^{2}=f(x)$ be a hyperelliptic curve over $\mathbb{Q}$ such that $\operatorname{deg} f$ is odd. For every place $v$ of $\mathbb{Q}$, any point on $J\left(\mathbb{Q}_{v}\right)$ can be represented by a divisor of degree 0 whose support is disjoint from the support of the divisor $\operatorname{div}(y)$. Then, we have $\delta_{v}(D)=1$ if $D$ is supported at $\infty$. If $D$ is of the form $D=\sum_{i=1}^{n} D_{i}$ with $D_{i}=\left(\alpha_{i}, 0\right)$, where $\alpha_{i}$ runs through all roots of a monic irreducible factor $h(x) \in \mathbb{Q}_{v}[x]$ of $f(x)$, then we have

$$
\delta_{v}(D)=(-1)^{\operatorname{deg} h}\left(h(x)-\frac{f(x)}{h(x)}\right) .
$$

For example, we obtain the following lemma.

Lemma 2.5. The following two elements of $L_{p}^{\times} / L_{p}^{\times 2}$ form an $\mathbb{F}_{2}$-basis of $\operatorname{Im}\left(\delta_{p}\right)$ :

$$
\left(2 ;-T_{2} ;-T_{3}\right), \quad\left(p ; T_{2} ; 2\right) .
$$

In particular, the following two elements of $I_{p}(L) / I_{p}(L)^{2}$ form an $\mathbb{F}_{2}$-basis of $G_{p}$ :

$$
\left((1) ;\left(T_{2}\right) ;\left(p, T_{3}\right)\right)_{p}, \quad\left((p) ;\left(T_{2}\right) ;(1)\right)_{p} .
$$

\footnotetext{
${ }^{6}$ The condition $p \equiv 3(\bmod 16)$ is used only to deduce that $-p$ is not square in $\mathbb{Q}_{v}$ for $v=2, p$. In fact, this lemma is true for $p \equiv 1,3,5(\bmod 8)$.
} 
Proof. Lemma 2.4 implies that

$$
\begin{aligned}
& \delta_{p}((0,0)-\infty)=-T+\left(T^{2}+p\right)\left(T^{2}+2 p\right)=\left(2 ;-T_{2} ;-T_{3}\right), \\
& \delta_{p}\left(\sum_{\substack{P \in C\left(\overline{\mathbb{Q}_{p}}\right) \\
x_{P}^{2}+p=0}} P-2 \infty\right)=\left(T^{2}+p\right)-T\left(T^{2}+2 p\right)=\left(p ; T_{2} ; 2\right) .
\end{aligned}
$$

Hence, $\left(2 ;-T_{2} ;-T_{3}\right),\left(p ; T_{2} ; 2\right) \in \operatorname{Im}\left(\delta_{p}\right)$.

By Lemma 2.3, it is sufficient to prove that the above two elements are linearly independent. Since $v_{T_{2}}\left(-T_{2}\right)=v_{T_{2}}\left(T_{2}\right)=1,\left(2 ;-T_{2} ;-T_{3}\right)$ and $\left(p ; T_{2} ; 2\right)$ are non-trivial in $L^{\times} / L^{\times 2}$. Moreover, since $v_{p}(2 p)=1,\left(2 ;-T_{2} ;-T_{3}\right)\left(p ; T_{2} ; 2\right)=\left(2 p ;-1 ;-2 T_{3}\right)$ is non-trivial in $L^{\times} / L^{\times 2}$. Thus, the lemma holds.

Lemma 2.6. (1) Suppose that $p \equiv 3(\bmod 32)$. Then, the following four elements of $L_{2}^{\times} / L_{2}^{\times 2}$ form an $\mathbb{F}_{2}$-basis of $\operatorname{Im}\left(\delta_{2}\right)$ :

$\left(2 ;-T_{2} ;-T_{3}\right), \quad\left(3 ; T_{2} ;-3\right), \quad\left(-1 ;-1-T_{2} ;-1-T_{3}\right), \quad\left(-1 ; 4+2 T_{2} ; 1+2 T_{3}\right)$.

(2) Suppose that $p \equiv 19(\bmod 32)$. Then, the following four elements of $L_{2}^{\times} / L_{2}^{\times 2}$ form an $\mathbb{F}_{2}$-basis of $\operatorname{Im}\left(\delta_{2}\right)$ :

$\left(2 ;-T_{2} ;-T_{3}\right), \quad\left(3 ; T_{2} ;-3\right), \quad\left(-3 ; 5-T_{2} ; 5-T_{3}\right), \quad\left(-1 ; 4+2 T_{2} ; 1+2 T_{3}\right)$.

In particular, the following two elements of $I_{2}(L) / I_{2}(L)^{2}$ form an $\mathbb{F}_{2}$-basis of $G_{2}$ :

$$
\left((2) ;(1) ;\left(2, T_{3}\right)\right)_{2}, \quad((1) ;(2) ;(1))_{2} \text {. }
$$

Proof. (1) First, we show that the above four elements actually lie in $\operatorname{Im}\left(\delta_{2}\right)$. Indeed, Lemma 2.4 implies that

$$
\begin{aligned}
& \delta_{2}((0,0)-\infty)=-T+\left(T^{2}+p\right)\left(T^{2}+2 p\right)=\left(2 ;-T_{2} ;-T_{3}\right), \\
& \delta_{2}\left(\sum_{\substack{P \in C\left(\overline{\mathbb{Q}_{2}}\right) \\
x_{P}^{2}+p=0}} P-2 \infty\right)=\left(T^{2}+p\right)-T\left(T^{2}+2 p\right)=\left(3 ; T_{2} ;-3\right) .
\end{aligned}
$$

Hence, $\left(2 ;-T_{2} ;-T_{3}\right),\left(3 ; T_{2} ;-3\right) \in \operatorname{Im}\left(\delta_{2}\right)$. Moreover, since $f(-1) \equiv 4(\bmod 32)$, there exists $Q \in C\left(\mathbb{Q}_{2}\right)$ such that $x_{Q}=-1$, and

$$
\left(-1 ;-1-T_{2} ;-1-T_{3}\right)=\delta_{2}(Q-\infty) \in \operatorname{Im}\left(\delta_{2}\right) .
$$

Finally, since $2=\tau^{2}$ in $\mathbb{Q}_{2}[\tau] /\left(\tau^{2}-2\right)$ and $p \equiv 1+\tau^{2}\left(\bmod \tau^{7}\right)$, we can check that

$$
f\left(1+\tau+\tau^{2}\right) \equiv\left(\tau+\tau^{2}+\tau^{3}\right)^{2} \quad\left(\bmod \tau^{7}\right),
$$

hence $f\left(1+\tau+\tau^{2}\right)$ is square in $\mathbb{Q}_{2}[\tau] /\left(\tau^{2}-2\right)$ by using Hensel's lemma. Therefore, we see that there exist $R, \bar{R} \in C\left(\overline{\mathbb{Q}_{2}}\right)$ such that $x_{R}+x_{\bar{R}}=6, x_{R} x_{\bar{R}}=7$, and $R+\bar{R}-2 \infty$ defines an element of $J\left(\mathbb{Q}_{2}\right)$. 7 Thus,

$$
\left(-1 ; 4+2 T_{2} ; 1+2 T_{3}\right)=\delta_{2}(R+\bar{R}-2 \infty) \in \operatorname{Im}\left(\delta_{2}\right) .
$$

By Lemma 2.3 , it is sufficient to prove that the above four elements are linearly independent. By taking their first components into account, we see that they are non-trivial in $L^{\times} / L^{\times 2}$, and the only possible relation is

$$
\left(-1 ;-1-T_{2} ;-1-T_{3}\right)\left(-1 ; 4+2 T_{2} ; 1+2 T_{3}\right)=\mathbf{1} .
$$

\footnotetext{
${ }^{7}$ We can take each element of $J\left(\mathbb{Q}_{2}\right)$ in one of the seven quadratic extensions of $\mathbb{Q}_{2}$.
} 
However, this is impossible because

$$
\left(-1-T_{3}\right)\left(1+2 T_{3}\right) \equiv 1+T_{3} \not \equiv 1 \quad\left(\bmod T_{3}^{2}\right) .
$$

Therefore, we obtain the assertion.

(2) The proof is similar to (1). Lemma 2.4 implies that $\left(2 ;-T_{2} ;-T_{3}\right),\left(3 ; T_{2} ;-3\right) \in$ $\operatorname{Im}\left(\delta_{2}\right)$. Since $f(5) \equiv 4(\bmod 32)$, there exists $Q \in C\left(\mathbb{Q}_{2}\right)$ such that $x_{Q}=5$, and $\left(-3 ; 5-T_{2} ; 5-T_{3}\right)=\delta_{2}(Q-\infty) \in \operatorname{Im}\left(\delta_{2}\right)$. Moreover, $\left(-1 ; 4+2 T_{2} ; 1+2 T_{3}\right) \in \operatorname{Im}\left(\delta_{2}\right)$ by the proof of (1).

By Lemma 2.3, it is sufficient to prove that the above four elements are linearly independent. By taking their first components into account, they are non-trivial in $L^{\times} / L^{\times 2}$, and only possible relation is

$$
\left(3 ; T_{2} ;-3\right)\left(-3 ; 5-T_{2} ; 5-T_{3}\right)\left(-1 ; 4+2 T_{2} ; 1+2 T_{3}\right)=\mathbf{1} .
$$

However, it is impossible because

$$
-p\left(5-T_{3}\right)\left(1+2 T_{3}\right) \equiv 1+T_{3} \not \equiv 1 \quad\left(\bmod T_{3}^{2}\right) .
$$

Therefore, we obtain the assertion.

By the first statement, to prove the second, it is sufficient to show that

$$
\left\{\begin{array}{l}
\operatorname{val}_{2}\left(2 ;-T_{2} ;-T_{3}\right)=\left((2) ;(1) ;\left(2, T_{3}\right)\right)_{2}, \\
\operatorname{val}_{2}\left(3 ; T_{2} ;-3\right)=\mathbf{1}_{2}, \\
\operatorname{val}_{2}\left(-1 ;-1-T_{2} ;-1-T_{3}\right)=((1) ;(2) ;(1))_{2}, \\
\operatorname{val}_{2}\left(-1 ; 4+2 T_{2} ; 1+2 T_{3}\right)=((1) ;(2) ;(1))_{2}, \\
\operatorname{val}_{2}\left(-3 ; 5-T_{2} ; 5-T_{3}\right)=((1) ;(2) ;(1))_{2} .
\end{array}\right.
$$

The first components are obvious. Since $p \equiv 3(\bmod 8)$, we can calculate the second components as follows.

- $v_{2}\left(T_{2}\right)=v_{2}\left(-T_{2}\right)=0$

- Since $v_{2}\left(N\left(-1-T_{2}\right)\right)=v_{2}(4)=2$ and $v_{2}\left(\operatorname{tr}\left(-1-T_{2}\right)\right)=v_{2}(-2)=1$, we have $v_{2}\left(-1-T_{2}\right)=1$.

- Since $v_{2}\left(N\left(4+2 T_{2}\right)\right)=v_{2}(28)=2$ and $v_{2}\left(\operatorname{tr}\left(4+2 T_{2}\right)\right)=v_{2}(8)=3$, we have $v_{2}\left(4+2 T_{2}\right)=1$.

- Since $v_{2}\left(N\left(5-T_{2}\right)\right)=v_{2}(28)=2$ and $v_{2}\left(\operatorname{tr}\left(5-T_{2}\right)\right)=v_{2}(10)=1$, we have $v_{2}\left(5-T_{2}\right)=1$.

Since $T_{3}$ is a uniformizer in $L_{2}^{(3)}$, we obtain the third components.

Remark 2.7. In fact, we can relate the above two cases in a more direct manner as follows: Let

$$
M_{2}:=\mathbb{Q}_{2}[\sigma] /\left(\sigma\left(\sigma^{2}+3\right)\left(\sigma^{2}+6\right)\right), \quad L_{2}:=\mathbb{Q}_{2}[\tau] /\left(\tau\left(\tau^{2}+19\right)\left(\tau^{2}+38\right)\right) .
$$

Then, we have an isomorphism $M_{2} \stackrel{\simeq}{\rightrightarrows} L_{2} ; \sigma \mapsto \tau / u^{2}$ of $\mathbb{Q}_{2}$-algebras, where $u \in \mathbb{Z}_{2}^{\times}$is taken so that $19=3 u^{4}$. It induces an isomorphism $M_{2}^{\times} / M_{2}^{\times 2} \stackrel{\widetilde{\Im}}{\rightarrow} L_{2}^{\times} / L_{2}^{\times 2}$ which maps $\left(-1 ;-1-\sigma_{2} ;-1-\sigma_{3}\right)$ to $-9-\tau=\left(3 ; \tau_{2} ;-3\right)\left(-3 ; 5-\tau_{2} ; 5-\tau_{3}\right)$, where $\sigma_{2}, \sigma_{3}$ (resp. $\tau_{2}$, $\left.\tau_{3}\right)$ are the images of $\sigma$ (resp. $\left.\tau\right)$ in $\mathbb{Q}_{2}[\sigma] /\left(\sigma^{2}+3\right), \mathbb{Q}_{2}[\sigma] /\left(\sigma^{2}+6\right)$ (resp. $\mathbb{Q}_{2}[\tau] /\left(\tau^{2}+19\right)$, $\left.\mathbb{Q}_{2}[\tau] /\left(\tau^{2}+38\right)\right)$.

The following proposition is essentially due to Gauss' genus theory.

Proposition 2.8 (cf. 8, Theorem 2], [3, Proposition 3.11, Theorem 5.30]). Let $K$ be an imaginary quadratic field of discriminant $D$.

(1) The class of an ideal $\mathfrak{a}$ of $K$ lies in $\mathrm{Cl}(K)^{2}$ if and only if the equation $N(\mathfrak{a}) x^{2}+$ $D y^{2}=z^{2}$ has a primitive $\mathbb{Z}_{l}$-solution for every prime number $l$ dividing $D$. 
(2) Let $r$ be the number of odd prime numbers dividing D. Then, we have

$$
\# \mathrm{Cl}(K)[2]=\left\{\begin{array}{lll}
2^{r-1} & D \equiv 1 \quad(\bmod 4), \\
2^{r} & D \equiv 0 & (\bmod 4) .
\end{array}\right.
$$

Corollary 2.9. The class of $\left(2, T_{3}\right)$ does not lie in $\mathrm{Cl}\left(L^{(3)}\right)^{2}$. In particular, $\mathrm{Cl}\left(L^{(3)}\right)[2]$ is generated by $\left(2, T_{3}\right)$.

Proof. By Proposition 2.8 (1), it is sufficient to prove that $2 x^{2}-8 p y^{2}=z^{2}$ has no primitive $\mathbb{Z}_{2}$-solution. We prove it by contradiction.

Suppose that $2 x^{2}-8 p y^{2}=z^{2}$ has a primitive $\mathbb{Z}_{2}$-solution $(x, y, z)$. Then, we have

- $x^{2}-4 p y^{2}=2 z^{\prime 2}$ for some $z^{\prime} \in \mathbb{Z}_{2}$, hence

- $2 x^{\prime 2}-2 p y^{2}=z^{\prime 2}$ for some $x^{\prime} \in \mathbb{Z}_{2}$, hence

- $x^{\prime 2}-p y^{2}=2 z^{\prime \prime 2}$ for some $z^{\prime \prime} \in \mathbb{Z}_{2}$.

Since $p \equiv 3(\bmod 8)$ and $y \in \mathbb{Z}_{2}^{\times}, x^{\prime 2}-p y^{2}$ is congruent to 1,5 or $6(\bmod 8)$. However, $2 z^{\prime \prime 2}$ is congruent to 0 or $2(\bmod 8)$, a contradiction. Therefore, the first statement follows. The second statement follows from Proposition $2.8(2)$ and $\left(2, T_{3}\right)^{2}=(2)$ as ideals.

Corollary 2.10. The following four elements of $L^{\times} / L^{\times 2}$ form an $\mathbb{F}_{2}$-basis of $\operatorname{Ker}(\mathrm{val})$ :

$$
(1 ; 1 ;-1), \quad(1 ;-1 ; 1), \quad(-1 ; 1 ; 1), \quad(1 ; 1 ; 2) .
$$

Proof. We have the following commutative diagram.

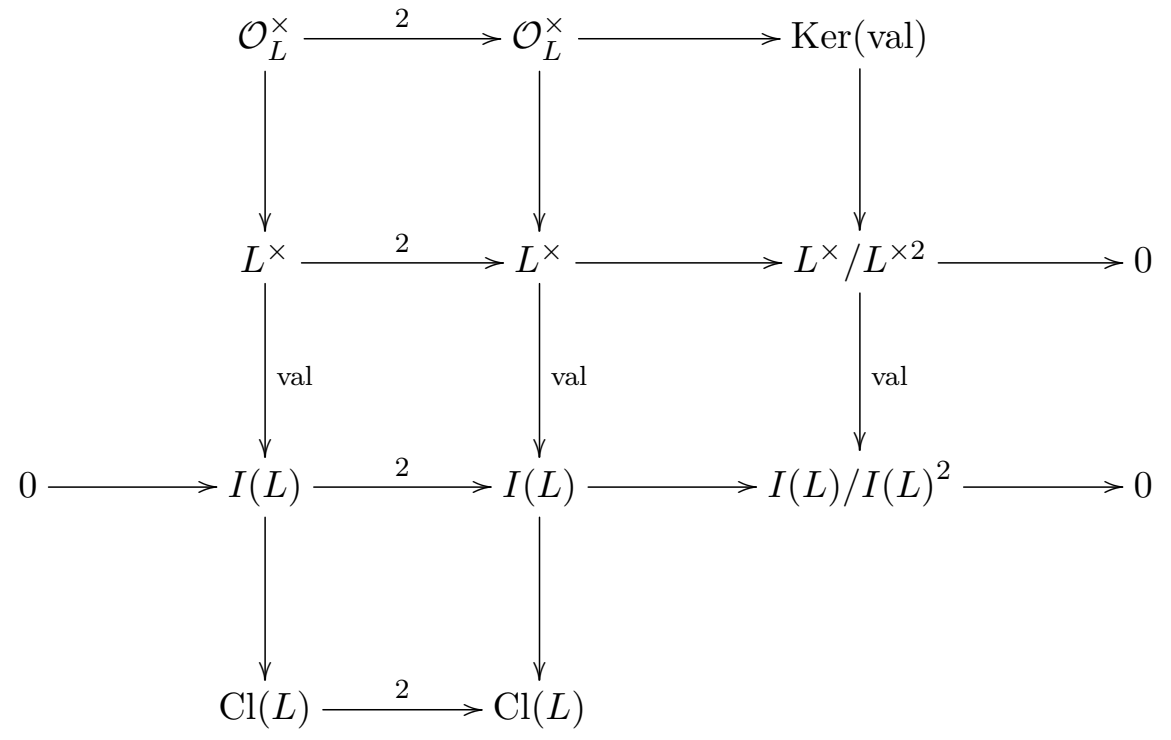

By applying the snake lemma, we obtain a short exact sequence

$$
1 \rightarrow \mathcal{O}_{L}^{\times} / \mathcal{O}_{L}^{\times 2} \rightarrow \operatorname{Ker}(\operatorname{val}) \rightarrow \mathrm{Cl}(L)[2] \rightarrow 0 .
$$

First, $(1 ; 1 ;-1),(1 ;-1 ; 1)$ and $(-1 ; 1 ; 1)$ form an $\mathbb{F}_{2}$-basis of $\mathcal{O}_{L}^{\times} / \mathcal{O}_{L}^{\times 2}$. Since the ring of integers of $L^{(1)} \simeq \mathbb{Q}$ is a PID, we have $\mathrm{Cl}\left(L^{(1)}\right)[2]=0$. By Proposition 2.8 (2), we have $\mathrm{Cl}\left(L^{(2)}\right)[2]=0$. Corollary 2.9 implies that $\mathrm{Cl}\left(L^{(3)}\right)[2]$ is generated by the class of $\left(2, T_{3}\right)$. Therefore, by a diagram chasing, we can check that the image of $(1 ; 1 ; 2)$ in $\mathrm{Cl}(L)[2]$ is non-trivial. This completes the proof.

Lemma 2.11. The following three elements of $I(L) / I(L)^{2}$ form an $\mathbb{F}_{2}$-basis of $W$ :

$$
((1) ;(2) ;(1))_{2} \times \mathbf{1}_{p}, \quad \mathbf{1}_{2} \times\left((p) ;\left(T_{2}\right) ;(1)\right)_{p}, \quad\left((2) ;(1) ;\left(2, T_{3}\right)\right)_{2} \times\left((1) ;\left(T_{2}\right) ;\left(p, T_{3}\right)\right)_{p} .
$$

Proof. By Lemmas 2.5 and 2.6 , the following four elements of $I(L) / I(L)^{2}$ form an $\mathbb{F}_{2}$-basis of $G$ :

$((1) ;(2) ;(1))_{2} \times \mathbf{1}_{p}, \quad\left((2) ;(1) ;\left(2, T_{3}\right)\right)_{2} \times \mathbf{1}_{p}, \quad \mathbf{1}_{2} \times\left((p) ;\left(T_{2}\right) ;(1)\right)_{p}, \quad \mathbf{1}_{2} \times\left((1) ;\left(T_{2}\right) ;\left(p, T_{3}\right)\right)_{p}$. 
Since the images of

$$
((1) ;(2) ;(1))_{2} \times \mathbf{1}_{p}, \quad \mathbf{1}_{2} \times\left((p) ;\left(T_{2}\right) ;(1)\right)_{p}, \quad\left((2) ;(1) ;\left(2, T_{3}\right)\right)_{2} \times\left((1) ;\left(T_{2}\right) ;\left(p, T_{3}\right)\right)_{p}
$$

in $\mathrm{Cl}(L)$ are trivial, the three elements in the statement lie in $W$. Moreover, we see that they are linearly independent. Finally, by Corollary 2.9 , we have $\left((2) ;(1) ;\left(2, T_{3}\right)\right)_{2} \times \mathbf{1}_{p} \notin$ $W$. Therefore, we obtain the assertion.

Lemma 2.12. The following seven elements of $L^{\times} / L^{\times 2}$ form an $\mathbb{F}_{2}$-basis of $\operatorname{val}^{-1}(G)$ :

$$
(1 ; 1 ;-1), \quad(1 ;-1 ; 1), \quad(-1 ; 1 ; 1), \quad(1 ; 1 ; 2), \quad(1 ; 2 ; 1), \quad\left(p ; T_{2} ; 1\right), \quad\left(2 ; T_{2} ; T_{3}\right) .
$$

Proof. By the definition of $W$, we have $\operatorname{val}\left(\operatorname{val}^{-1}(G)\right) \subset W$. Moreover, we have $W \subset$ $\operatorname{val}\left(\operatorname{val}^{-1}(G)\right)$ : Indeed, for every $[\mathfrak{a}] \in W$, there exists $\mathfrak{b} \in I(L)$ such that $\left[\mathfrak{a} \mathfrak{b}^{2}\right]=[(1)]$ in $\mathrm{Cl}(L) / \mathrm{Cl}(L)^{2}$. Thus, there exists $a \in L^{\times}$such that $[\mathfrak{a}]=\left[\mathfrak{a b}^{2}\right]=[(a)]$ in $I(L) / I(L)^{2}$. Therefore, we obtain the following short exact sequence:

$$
0 \rightarrow \operatorname{Ker}(\mathrm{val}) \rightarrow \mathrm{val}^{-1}(G) \stackrel{\mathrm{val}}{\rightarrow} W \rightarrow 0
$$

By Corollary 2.10 and Lemma 2.11, we obtain the assertion.

Lemma 2.13. Set elements of $L_{2}^{\times} / L_{2}^{\times 2} \times L_{p}^{\times} / L_{p}^{\times 2}$ as follows:

$$
\begin{aligned}
& d_{1}:=\left(2 ;-T_{2} ;-T_{3}\right)_{2} \times \mathbf{1}_{p}, \\
& d_{2}:=\left(3 ; T_{2} ;-3\right)_{2} \times \mathbf{1}_{p}, \\
& d_{3}:=\left(-1 ;-1-T_{2} ;-1-T_{3}\right)_{2} \times \mathbf{1}_{p}, \quad \tilde{d}_{3}:=\left(-3 ; 5-T_{2} ; 5-T_{3}\right)_{2} \times \mathbf{1}_{p}, \\
& d_{4}:=\left(-1 ; 4+2 T_{2} ; 1+2 T_{3}\right)_{2} \times \mathbf{1}_{p}, \\
& d_{5}:=\mathbf{1}_{2} \times\left(2 ;-T_{2} ;-T_{3}\right)_{p}, \\
& d_{6}:=\mathbf{1}_{2} \times\left(p ; T_{2} ; 2\right)_{p}, \\
& h_{1}:=\operatorname{res}_{S}(1 ; 1 ;-1)=(1 ; 1 ;-1)_{2} \times(1 ; 1 ;-1)_{p}, \\
& h_{2}:=\operatorname{res}_{S}(1 ;-1 ; 1)=(1 ;-1 ; 1)_{2} \times(1 ;-1 ; 1)_{p}, \\
& h_{3}:=\operatorname{res}_{S}(-1 ; 1 ; 1)=(-1 ; 1 ; 1)_{2} \times(-1 ; 1 ; 1)_{p}, \\
& h_{4}:=\operatorname{res}_{S}(1 ; 1 ; 2)=(1 ; 1 ; 2)_{2} \times(1 ; 1 ; 2)_{p}, \\
& h_{5}:=\operatorname{res}_{S}(1 ; 2 ; 1)=(1 ; 2 ; 1)_{2} \times(1 ; 2 ; 1)_{p}, \\
& h_{6}:=\operatorname{res}_{S}\left(p ; T_{2} ; 1\right)=\left(p ; T_{2} ; 1\right)_{2} \times\left(p ; T_{2} ; 1\right)_{p}, \\
& h_{7}:=\operatorname{res}_{S}\left(2 ; T_{2} ; T_{3}\right)=\left(2 ; T_{2} ; T_{3}\right)_{2} \times\left(2 ; T_{2} ; T_{3}\right)_{p} .
\end{aligned}
$$

(1) We have $d_{1} d_{5} h_{1} h_{2} h_{7}=d_{2} d_{6} h_{4} h_{6}=\mathbf{1}_{2} \times \mathbf{1}_{p}$.

(2) Suppose that $p \equiv 3(\bmod 32)$. Then $, d_{1}, d_{2}, d_{3}, d_{4}, h_{1}, \ldots, h_{7}$ form an $\mathbb{F}_{2}$-basis of $\left(\operatorname{Im}\left(\delta_{2}\right) \times \operatorname{Im}\left(\delta_{p}\right)\right)+\operatorname{res}_{S}\left(\mathrm{val}^{-1}(G)\right)$.

(3) Suppose that $p \equiv 19(\bmod 32)$. Then $, d_{1}, d_{2}, \tilde{d}_{3}, d_{4}, h_{1}, \ldots, h_{7}$ form an $\mathbb{F}_{2}$-basis of $\left(\operatorname{Im}\left(\delta_{2}\right) \times \operatorname{Im}\left(\delta_{p}\right)\right)+\operatorname{res}_{S}\left(\operatorname{val}^{-1}(G)\right)$.

Proof. (1) We can check it by direct calculation. 
(2) By (1), Lemmas 2.5, 2.6 and 2.12, $\left(\operatorname{Im}\left(\delta_{2}\right) \times \operatorname{Im}\left(\delta_{p}\right)\right)+\operatorname{res}_{S}\left(\right.$ val $\left.^{-1}(G)\right)$ is generated by $d_{1}, \ldots d_{4}, h_{1}, \ldots, h_{7}$. Set

$$
\begin{aligned}
& d_{1}=\left(2 ;-T_{2} ;-T_{3}\right)_{2} \times \mathbf{1}_{p}, \\
& d_{2}=\left(3 ; T_{2} ;-3\right)_{2} \times \mathbf{1}_{p}, \\
& d_{3}^{\prime}:=d_{3} d_{4}=\left(1 ;\left(-1-T_{2}\right)\left(4+2 T_{2}\right) ;\left(-1-T_{3}\right)\left(1+2 T_{3}\right)\right)_{2} \times \mathbf{1}_{p}, \\
& d_{4}=\left(-1 ; 4+2 T_{2} ; 1+2 T_{3}\right)_{2} \times \mathbf{1}_{p}, \\
& h_{1}^{\prime}:=h_{1} h_{4}=(1 ; 1 ;-2)_{2} \times \mathbf{1}_{p}, \\
& h_{2}^{\prime}:=h_{2} h_{5}=(1 ;-2 ; 1)_{2} \times \mathbf{1}_{p}, \\
& h_{3}^{\prime}:=h_{3} h_{7}=\left(-2 ; T_{2} ; T_{3}\right)_{2} \times\left(1 ; T_{2} ; T_{3}\right)_{p}, \\
& h_{4}=\operatorname{res}_{S}(1 ; 1 ; 2)=(1 ; 1 ; 2)_{2} \times(1 ; 1 ; 2)_{p}, \\
& h_{5}=\operatorname{res}_{S}(1 ; 2 ; 1)=(1 ; 2 ; 1)_{2} \times(1 ; 2 ; 1)_{p}, \\
& h_{6}=\operatorname{res}_{S}\left(p ; T_{2} ; 1\right)=\left(p ; T_{2} ; 1\right)_{2} \times\left(p ; T_{2} ; 1\right)_{p}, \\
& h_{7}=\operatorname{res}_{S}\left(2 ; T_{2} ; T_{3}\right)=\left(2 ; T_{2} ; T_{3}\right)_{2} \times\left(2 ; T_{2} ; T_{3}\right)_{p} .
\end{aligned}
$$

It is sufficient to prove that the above eleven elements are linearly independent.

(a) By taking the first components at $v=p$ into account, we see that there is no relation containing $h_{6}$ and $h_{7}$.

(b) By taking the second components at $v=p$ into account, we see that there is no relation containing $h_{3}^{\prime}$ and $h_{5}$.

(c) By taking the third components at $v=p$ into account, we see that there is no relation containing $h_{4}$.

(d) By taking the first components at $v=2$ into account, we see that there is no relation containing $d_{1}, d_{2}$ and $d_{4}$.

(e) By taking the third components at $v=2$ into account, we see that there is no relation containing $d_{3}^{\prime}$ and $h_{1}^{\prime}$ because

- $\left(-1-T_{3}\right)\left(1+2 T_{3}\right) \equiv 1+T_{3}\left(\bmod T_{3}^{2}\right)$,

- $-2 \equiv T_{3}^{2}\left(1+T_{3}^{2}\right)\left(\bmod T_{3}^{6}\right)$,

- $-2\left(-1-T_{3}\right)\left(1+2 T_{3}\right) \equiv T_{3}^{2}\left(1+T_{3}\right)\left(\bmod T_{3}^{4}\right)$.

(f) Finally, by taking the second components at $v=2$ into account, we see that there is no relation containing $h_{2}^{\prime}$.

(3) Respectively, (2) holds.

By (1), Lemmas 2.5. 2.6 and 2.12, $\left(\operatorname{Im}\left(\delta_{2}\right) \times \operatorname{Im}\left(\delta_{p}\right)\right)+\operatorname{res}_{S}\left(\operatorname{val}^{-1}(G)\right)$ is generated by $d_{1}, \ldots d_{4}, h_{1}, \ldots, h_{7}$. Set

$$
\begin{aligned}
& d_{1}=\left(2 ;-T_{2} ;-T_{3}\right)_{2} \times \mathbf{1}_{p}, \\
& d_{2}^{\prime}:=d_{2} d_{3} d_{4}=\left(1 ; T_{2}\left(5-T_{2}\right)\left(4+2 T_{2}\right) ;-p\left(5-T_{3}\right)\left(1+2 T_{3}\right)\right)_{2} \times \mathbf{1}_{p}, \\
& \tilde{d}_{3}=\left(-3 ; 5-T_{2} ; 5-T_{3}\right)_{2} \times \mathbf{1}_{p}, \\
& d_{4}=\left(-1 ; 4+2 T_{2} ; 1+2 T_{3}\right)_{2} \times \mathbf{1}_{p}, \\
& h_{1}^{\prime}:=h_{1} h_{4}=(1 ; 1 ;-2)_{2} \times \mathbf{1}_{p}, \\
& h_{2}^{\prime}:=h_{2} h_{5}=(1 ;-2 ; 1)_{2} \times \mathbf{1}_{p}, \\
& h_{3}^{\prime}:=h_{3} h_{7}=\left(-2 ; T_{2} ; T_{3}\right)_{2} \times\left(1 ; T_{2} ; T_{3}\right)_{p}, \\
& h_{4}=\operatorname{res}_{S}(1 ; 1 ; 2)=(1 ; 1 ; 2)_{2} \times(1 ; 1 ; 2)_{p}, \\
& h_{5}=\operatorname{res}_{S}(1 ; 2 ; 1)=(1 ; 2 ; 1)_{2} \times(1 ; 2 ; 1)_{p}, \\
& h_{6}=\operatorname{res}_{S}\left(p ; T_{2} ; 1\right)=\left(p ; T_{2} ; 1\right)_{2} \times\left(p ; T_{2} ; 1\right)_{p}, \\
& h_{7}=\operatorname{res}_{S}\left(2 ; T_{2} ; T_{3}\right)=\left(2 ; T_{2} ; T_{3}\right)_{2} \times\left(2 ; T_{2} ; T_{3}\right)_{p} .
\end{aligned}
$$

It is sufficient to prove that the above eleven elements are linearly independent. 
(a) By taking the first components at $v=p$ into account, we see that there is no relation containing $h_{6}$ and $h_{7}$.

(b) By taking the second components at $v=p$ into account, we see that there is no relation containing $h_{3}^{\prime}$ and $h_{5}$.

(c) By taking the third components at $v=p$ into account, we see that there is no relation containing $h_{4}$.

(d) By taking the first components at $v=2$ into account, we see that there is no relation containing $d_{1}, \tilde{d}_{3}$ and $d_{4}$.

(e) By taking the third components at $v=2$ into account, we see that there is no relation containing $d_{2}^{\prime}$ and $h_{1}^{\prime}$ because

- $-p\left(5-T_{3}\right)\left(1+2 T_{3}\right) \equiv 1+T_{3}\left(\bmod T_{3}^{2}\right)$,

- $-2 \equiv T_{3}^{2}\left(1+T_{3}^{2}\right)\left(\bmod T_{3}^{6}\right)$,

- Since $2 p\left(5-T_{3}\right)\left(1+2 T_{3}\right) \equiv T_{3}^{2}\left(1+T_{3}\right)\left(\bmod T_{3}^{4}\right)$.

(f) Finally, by taking the second components at $v=2$ into account, we see that there is no relation containing $h_{2}^{\prime}$.

Recall that

$$
\begin{aligned}
\operatorname{dim} \operatorname{Sel}^{(2)}\left(\mathbb{Q}, J^{(p ; i, j)}\right)= & \operatorname{dim} \operatorname{val}^{-1}(G)+\operatorname{dim}\left(\operatorname{Im}\left(\delta_{2}\right) \times \operatorname{Im}\left(\delta_{p}\right)\right) \\
& -\operatorname{dim}\left(\left(\operatorname{Im}\left(\delta_{2}\right) \times \operatorname{Im}\left(\delta_{p}\right)\right)+\operatorname{res}_{S}\left(\operatorname{val}^{-1}(G)\right)\right)
\end{aligned}
$$

and

$$
J(\mathbb{Q}) / 2 J(\mathbb{Q}) \simeq \mathbb{F}_{2}^{\operatorname{rank}(J(\mathbb{Q}))} \oplus J(\mathbb{Q})[2] .
$$

Therefore, by Lemmas 2.3, 2.5, 2.6, 2.12 and 2.13, we obtain

$$
\operatorname{rank}(J(\mathbb{Q})) \leq \operatorname{dim} \operatorname{Sel}^{(2)}(\mathbb{Q}, J)-\operatorname{dim} J(\mathbb{Q})[2]=7+6-11-2=0 .
$$

This completes the proof of Theorem 2.1 (1).

2.2. Case $(i, j)=(1,1)$

Suppose that $p \equiv 11(\bmod 16)$. Then, we have the following irreducible decompositions:

$$
\left\{\begin{array}{l}
L_{2}=\mathbb{Q}_{2}\left[T_{1}\right] /\left(T_{1}\right) \times \mathbb{Q}_{2}\left[T_{2}\right] /\left(T_{2}^{2}+2 p\right) \times \mathbb{Q}_{2}\left[T_{3}\right] /\left(T_{3}^{2}+4 p\right), \\
L_{p}=\mathbb{Q}_{p}\left[T_{1}\right] /\left(T_{1}\right) \times \mathbb{Q}_{p}\left[T_{2}\right] /\left(T_{2}^{2}+2 p\right) \times \mathbb{Q}_{p}\left[T_{3}\right] /\left(T_{3}^{2}+4 p\right) .
\end{array}\right.
$$

Lemma 2.14. The following two elements form an $\mathbb{F}_{2}$-basis of $J(\mathbb{Q})[2]$ and $J\left(\mathbb{Q}_{v}\right)[2]$ for $v=2, p, \infty$ :

$$
(0,0)-\infty, \quad \sum_{\substack{P \in C(\overline{\mathbb{Q}}) \\ x_{P}^{2}+2 p=0}} P-2 \infty .
$$

In particular, we have the following table.

\begin{tabular}{|l||l|l|}
\hline$l$ & $\operatorname{dim} J\left(\mathbb{Q}_{v}\right)[2]$ & $\operatorname{dim} \delta_{v}\left(J\left(\mathbb{Q}_{v}\right)\right)$ \\
\hline \hline 2 & 2 & 4 \\
\hline$p$ & 2 & 2 \\
\hline$\infty$ & 2 & 0 \\
\hline
\end{tabular}

Proof. The first statement follows from [12, Lemma 5.2]. Note that neither $-p$ nor $-2 p$ is not square in $\mathbb{Q}_{v}$ for $v=2, p, \infty$. The second statement follows from the following formula 
(cf. 4, p. 451, proof of Lemma 3]).

$$
\operatorname{dim}_{\mathbb{F}_{2}} \delta_{v}\left(J\left(\mathbb{Q}_{v}\right)\right)=\operatorname{dim}_{\mathbb{F}_{2}} J\left(\mathbb{Q}_{v}\right)[2] \begin{cases}+0 & (v \neq 2, \infty), \\ +2 & (v=2), \\ -2 & (v=\infty)\end{cases}
$$

Lemma 2.15. The following two elements of $L_{p}^{\times} / L_{p}^{\times 2}$ form an $\mathbb{F}_{2}$-basis of $\operatorname{Im}\left(\delta_{p}\right)$ :

$$
\left(2 ;-T_{2} ;-T_{3}\right), \quad\left(2 p ; T_{2} ; 2\right) .
$$

In particular, the following two elements of $I_{p}(L) / I_{p}(L)^{2}$ form an $\mathbb{F}_{2}$-basis of $G_{p}$ :

$$
\left((1) ;\left(p, T_{2}\right) ;\left(T_{3}\right)\right)_{p}, \quad\left((p) ;\left(p, T_{2}\right) ;(1)\right)_{p} .
$$

Proof. Lemma 2.4 implies that

$$
\begin{aligned}
& \delta_{p}((0,0)-\infty)=-T+\left(T^{2}+2 p\right)\left(T^{2}+4 p\right)=\left(2 ;-T_{2} ;-T_{3}\right), \\
& \delta_{p}\left(\sum_{\substack{P \in C(\overline{\mathbb{Q} p}) \\
x_{P}^{2}+2 p=0}} P-2 \infty\right)=\left(T^{2}+2 p\right)-T\left(T^{2}+4 p\right)=\left(2 p ; T_{2} ; 2\right) .
\end{aligned}
$$

Hence, $\left(2 ;-T_{2} ;-T_{3}\right),\left(2 p ; T_{2} ; 2\right) \in \operatorname{Im}\left(\delta_{p}\right)$.

By Lemma 2.14, it is sufficient to prove that the above two elements are linearly independent. Since $v_{T_{3}}\left(-T_{3}\right)=1$ and $v_{p}(2 p)=1,\left(2 ;-T_{2} ;-T_{3}\right)$ and $\left(2 p ; T_{2} ; 2\right)$ are non-trivial in $L^{\times} / L^{\times 2}$. Moreover, since $v_{p}(p)=1,\left(2 ;-T_{2} ;-T_{3}\right)\left(2 p ; T_{2} ; 2\right)=\left(p ;-1 ;-2 T_{3}\right)$ is non-trivial in $L^{\times} / L^{\times 2}$. Thus, the lemma holds.

Lemma 2.16. The following four elements of $L_{2}^{\times} / L_{2}^{\times 2}$ form an $\mathbb{F}_{2}$-basis of $\operatorname{Im}\left(\delta_{2}\right)$ :

$$
\left(2 ;-T_{2} ;-T_{3}\right), \quad\left(6 ; T_{2} ; 2\right), \quad\left(3 ; 3-T_{2} ; 3-T_{3}\right), \quad\left(5 ; 3 ; 2-2 T_{3}\right) .
$$

In particular, the following two elements of $I_{2}(L) / I_{2}(L)^{2}$ form an $\mathbb{F}_{2}$-basis of $G_{2}$ :

$$
\left((2) ;\left(2, T_{2}\right) ;(2)\right)_{2}, \quad((1) ;(1) ;(2))_{2} .
$$

Proof. First, we show that the four elements in the statement actually lie in $\operatorname{Im}\left(\delta_{2}\right)$. Indeed, Lemma 2.4 implies that

$$
\begin{aligned}
& \delta_{2}((0,0)-\infty)=-T+\left(T^{2}+2 p\right)\left(T^{2}+4 p\right)=\left(2 ;-T_{2} ;-T_{3}\right), \\
& \delta_{2}\left(\sum_{\substack{P \in C(\overline{\mathbb{Q}}) \\
x_{P}^{2}+2 p=0}} P-2 \infty\right)=\left(T^{2}+2 p\right)-T\left(T^{2}+4 p\right)=\left(6 ; T_{2} ; 2\right) .
\end{aligned}
$$

Hence, $\left(2 ;-T_{2} ;-T_{3}\right),\left(6 ; T_{2} ; 2\right) \in \operatorname{Im}\left(\delta_{2}\right)$. Moreover, since $f(3) \equiv 1(\bmod 8)$, there exists $Q \in C\left(\mathbb{Q}_{2}\right)$ such that $x_{Q}=3$, and

$$
\left(3 ; 3-T_{2} ; 3-T_{3}\right)=\delta_{2}(Q-\infty) \in \operatorname{Im}\left(\delta_{2}\right) .
$$

Finally, since $2=\tau^{2}+\tau^{3}$ in $\mathbb{Q}_{2}[\tau] /\left(\tau^{2}+2 \tau+2\right)$ and $p \equiv 1+\tau^{2}+\tau^{3}+\tau^{6}\left(\bmod \tau^{7}\right)$, we can check that $f\left(\tau^{2}\left(1+\tau^{2}\right)\right) \equiv \tau^{10}(1+\tau)^{2}\left(1+\tau^{2}\right)^{2}\left(\bmod \tau^{15}\right)$, hence $f\left(\tau^{2}\left(1+\tau^{2}\right)\right)$ is square in $\mathbb{Q}_{2}[\tau] /\left(\tau^{2}+2 \tau+2\right)$ by using Hensel's lemma. Therefore, we see that there exist $R, \bar{R}$ such that $x_{R}+x_{\bar{R}}=-8, x_{R} x_{\bar{R}}=20$, and $R+\bar{R}-2 \infty$ defines an element in $J\left(\mathbb{Q}_{2}\right)$. Thus,

$$
\left(5 ; 3 ; 2-2 T_{3}\right)=\delta_{2}(R+\bar{R}-2 \infty) \in \operatorname{Im}\left(\delta_{2}\right) .
$$


By Lemma 2.14, it is sufficient to prove that the four elements in the statement are linearly independent. By taking their first components into account, the four elements in the statement are non-trivial in $\operatorname{Im}\left(\delta_{2}\right)$, and the only possible relation is

$$
\left(2 ;-T_{2} ;-T_{3}\right)\left(6 ; T_{2} ; 2\right)\left(3 ; 3-T_{2} ; 3-T_{3}\right)=\mathbf{1} .
$$

However, this is impossible because

$$
T_{2}-3 \equiv 1+T_{2} \not \equiv 1 \quad\left(\bmod T_{2}^{2}\right) .
$$

Therefore, we obtain the assertion.

By the first statement, to prove the second, it is sufficient to show that

$$
\left\{\begin{array}{l}
\operatorname{val}_{2}\left(2 ;-T_{2} ;-T_{3}\right)=\left((2) ;\left(2, T_{2}\right) ;(2)\right)_{2}, \\
\operatorname{val}_{2}\left(6 ; T_{2} ; 2\right)=\left((2) ;\left(2, T_{2}\right) ;(2)\right)_{2} \\
\operatorname{val}_{2}\left(3 ; 3-T_{2} ; 3-T_{3}\right)=\mathbf{1}_{2} \\
\operatorname{val}_{2}\left(5 ; 3 ; 2-2 T_{3}\right)=((1) ;(1) ;(2))_{2}
\end{array}\right.
$$

First, the first components are obvious. Since $T_{2}$ is a uniformizer in $L_{2}^{(2)}$, we obtain the second components. Since 2 is a uniformizer in $L_{2}^{(3)}$ we obtain the third components.

Corollary 2.17. The following four elements of $L^{\times} / L^{\times 2}$ form an $\mathbb{F}_{2}$-basis of $\operatorname{Ker}(\mathrm{val})$ :

$$
(1 ; 1 ;-1), \quad(1 ;-1 ; 1), \quad(-1 ; 1 ; 1), \quad(1 ; 2 ; 1) .
$$

Proof. By the exactly same manner as in Corollary 2.10, we obtain a short exact sequence

$$
1 \rightarrow \mathcal{O}_{L}^{\times} / \mathcal{O}_{L}^{\times 2} \rightarrow \operatorname{Ker}(\operatorname{val}) \rightarrow \mathrm{Cl}(L)[2] \rightarrow 0 .
$$

First, $(1 ; 1 ;-1),(1 ;-1 ; 1)$ and $(-1 ; 1 ; 1)$ form an $\mathbb{F}_{2}$-basis of $\mathcal{O}_{L}^{\times} / \mathcal{O}_{L}^{\times 2}$. Since the ring of integers of $L^{(1)} \simeq \mathbb{Q}$ is a PID, we have $\operatorname{Cl}\left(L^{(1)}\right)[2]=0$. Corollary 2.9 implies that $\operatorname{Cl}\left(L^{(2)}\right)[2]$ is generated by the class of $\left(2, T_{2}\right)$. By Proposition $2.8(2)$, we have $\mathrm{Cl}\left(L^{(3)}\right)[2]=0$. Therefore, by a diagram chasing, we can check that the image of $(1 ; 2 ; 1)$ in $\mathrm{Cl}(L)[2]$ is non-trivial. This completes the proof.

Lemma 2.18. The following three elements of $I(L) / I(L)^{2}$ form an $\mathbb{F}_{2}$-basis of $W$ :

$$
((1) ;(1) ;(2))_{2} \times \mathbf{1}_{p}, \quad\left((2) ;\left(2, T_{2}\right) ;(2)\right)_{2} \times\left((1) ;\left(p, T_{2}\right) ;\left(T_{3}\right)\right)_{p}, \quad \mathbf{1}_{2} \times\left((p) ;(1) ;\left(T_{3}\right)\right)_{p} .
$$

Proof. Lemmas 2.15 and 2.16 , the following four elements of $I(L) / I(L)^{2}$ form an $\mathbb{F}_{2}$-basis of $G$.

$$
\begin{array}{rr}
\left((2) ;\left(2, T_{2}\right) ;(2)\right)_{2} \times \mathbf{1}_{p}, & ((1) ;(1) ;(2))_{2} \times \mathbf{1}_{p}, \\
\mathbf{1}_{2} \times\left((1) ;\left(p, T_{2}\right) ;\left(T_{3}\right)\right)_{p}, & \mathbf{1}_{2} \times\left((p) ;\left(p, T_{2}\right) ;(1)\right)_{p} .
\end{array}
$$

Since the images of

$$
((1) ;(1) ;(2))_{2} \times \mathbf{1}_{p}, \quad\left((2) ;\left(2, T_{2}\right) ;(2)\right)_{2} \times\left((1) ;\left(p, T_{2}\right) ;\left(T_{3}\right)\right)_{p}, \quad \mathbf{1}_{2} \times\left((p) ;(1) ;\left(T_{3}\right)\right)_{p} .
$$

in $\mathrm{Cl}(L)$ are trivial, the three elements in the statement lie in $W$. Moreover, we see that they are linearly independent. Finally, by Corollary 2.9 , we have $\left((2) ;\left(2, T_{2}\right) ;(2)\right)_{2} \times \mathbf{1}_{p} \notin$ $W$. Therefore, we obtain the assertion.

By the exactly same manner as in Lemma 2.12, the following lemma follows from Corollary 2.17 and Lemma 2.18 .

Lemma 2.19. The following seven elements of $L^{\times} / L^{\times 2}$ form an $\mathbb{F}_{2}$-basis of $\operatorname{val}^{-1}(G)$ :

$$
(1 ; 1 ;-1), \quad(1 ;-1 ; 1), \quad(-1 ; 1 ; 1), \quad(1 ; 2 ; 1), \quad(1 ; 1 ; 2), \quad\left(2 ; T_{2} ; T_{3}\right), \quad\left(p ; 1 ; T_{3}\right) .
$$


Lemma 2.20. Set elements of $L_{2}^{\times} / L_{2}^{\times 2} \times L_{p}^{\times} / L_{p}^{\times 2}$ as follows:

$$
\begin{aligned}
& d_{1}:=\left(2 ;-T_{2} ;-T_{3}\right)_{2} \times \mathbf{1}_{p}, \\
& d_{2}:=\left(6 ; T_{2} ; 2\right)_{2} \times \mathbf{1}_{p}, \\
& d_{3}:=\left(3 ; 3-T_{2} ; 3-T_{3}\right)_{2} \times \mathbf{1}_{p}, \\
& d_{4}:=\left(5 ; 3 ; 2-2 T_{3}\right)_{2} \times \mathbf{1}_{p}, \\
& d_{5}:=\mathbf{1}_{2} \times\left(2 ;-T_{2} ;-T_{3}\right)_{p}, \\
& d_{6}:=\mathbf{1}_{2} \times\left(2 p ; T_{2} ; 2\right)_{p}, \\
& h_{1}:=\operatorname{res}_{S}(1 ; 1 ;-1)=(1 ; 1 ;-1)_{2} \times(1 ; 1 ;-1)_{p}, \\
& h_{2}:=\operatorname{res}_{S}(1 ;-1 ; 1)=(1 ;-1 ; 1)_{2} \times(1 ;-1 ; 1)_{p}, \\
& h_{3}:=\operatorname{res}_{S}(-1 ; 1 ; 1)=(-1 ; 1 ; 1)_{2} \times(-1 ; 1 ; 1)_{p}, \\
& h_{4}:=\operatorname{res}_{S}(1 ; 2 ; 1)=(1 ; 2 ; 1)_{2} \times(1 ; 2 ; 1)_{p}, \\
& h_{5}:=\operatorname{res}_{S}(1 ; 1 ; 2)=(1 ; 1 ; 2)_{2} \times(1 ; 1 ; 2)_{p}, \\
& h_{6}:=\operatorname{res}_{S}\left(2 ; T_{2} ; T_{3}\right)=\left(2 ; T_{2} ; T_{3}\right)_{2} \times\left(2 ; T_{2} ; T_{3}\right)_{p}, \\
& h_{7}:=\operatorname{res}_{S}\left(p ; 1 ; T_{3}\right)=\left(3 ; 1 ; T_{3}\right)_{2} \times\left(p ; 1 ; T_{3}\right)_{p} .
\end{aligned}
$$

(1) We have $d_{1} d_{5} h_{1} h_{2} h_{6}=d_{2} d_{6} h_{7}=\mathbf{1}_{2} \times \mathbf{1}_{p}$.

(2) $d_{1}, \ldots, d_{4}, h_{1}, \ldots, h_{7}$ form an $\mathbb{F}_{2}$-basis of $\left(\operatorname{Im}\left(\delta_{2}\right) \times \operatorname{Im}\left(\delta_{p}\right)\right)+\operatorname{res}_{S}\left(\operatorname{val}^{-1}(G)\right)$.

Proof. (1) We can check it by direct calculation.

(2) By (1), Lemmas 2.15, 2.16 and 2.19 $\left(\operatorname{Im}\left(\delta_{2}\right) \times \operatorname{Im}\left(\delta_{p}\right)\right)+\operatorname{res}_{S}\left(\right.$ val $\left.^{-1}(G)\right)$ is generated by $d_{1}, \ldots d_{4}, h_{1}, \ldots, h_{7}$. Set

$$
\begin{aligned}
& d_{1}^{\prime}:=d_{1} d_{2} d_{3}=\left(1 ;-3+T_{2} ; 2 T_{3}\left(T_{3}-3\right)\right)_{2} \times \mathbf{1}_{p}, \\
& d_{2}=\left(6 ; T_{2} ; 2\right)_{2} \times \mathbf{1}_{p}, \\
& d_{3}=\left(3 ; 3-T_{2} ; 3-T_{3}\right)_{2} \times \mathbf{1}_{p}, \\
& d_{4}=\left(5 ; 3 ; 2-2 T_{3}\right)_{2} \times \mathbf{1}_{p}, \\
& h_{1}^{\prime}:=h_{1} h_{5}=(1 ; 1 ;-2)_{2} \times \mathbf{1}_{p}, \\
& h_{2}^{\prime}:=h_{2} h_{4}=(1 ;-2 ; 1)_{2} \times \mathbf{1}_{p}, \\
& h_{3}^{\prime}:=h_{3} h_{6}=\left(-2 ; T_{2} ; T_{3}\right)_{2} \times\left(1 ; T_{2} ; T_{3}\right)_{p}, \\
& h_{4}=\operatorname{res}_{S}(1 ; 2 ; 1)=(1 ; 2 ; 1)_{2} \times(1 ; 2 ; 1)_{p}, \\
& h_{5}=\operatorname{res}_{S}(1 ; 1 ; 2)=(1 ; 1 ; 2)_{2} \times(1 ; 1 ; 2)_{p}, \\
& h_{6}=\operatorname{res}_{S}\left(2 ; T_{2} ; T_{3}\right)=\left(2 ; T_{2} ; T_{3}\right)_{2} \times\left(2 ; T_{2} ; T_{3}\right)_{p}, \\
& h_{7}:=\operatorname{res}_{S}\left(p ; 1 ; T_{3}\right)=\left(3 ; 1 ; T_{3}\right)_{2} \times\left(p ; 1 ; T_{3}\right)_{p} .
\end{aligned}
$$

It is sufficient to prove that the above eleven elements are linearly independent.

(a) By taking the first components at $v=p$ into account, we see that there is no relation containing $h_{6}$ and $h_{7}$.

(b) By taking the second components at $v=p$ into account, we see that there is no relation containing $h_{3}^{\prime}$ and $h_{4}$.

(c) By taking the third components at $v=p$ into account, we see that there is no relation containing $h_{5}$.

(d) By taking the first components at $v=2$ into account, we see that there is no relation containing $d_{2}, d_{3}$ and $d_{4}$.

(e) By taking the second components at $v=2$ into account, we see that there is no relation containing $d_{1}^{\prime}$ and $h_{2}^{\prime}$ because

- $-3+T_{2} \equiv T_{2}+1\left(\bmod T_{2}^{2}\right)$,

- -2 is non-square in $L_{2}^{(2)} \simeq \mathbb{Q}_{2}(\sqrt{-6})$, 
- $-2\left(-3+T_{2}\right) \equiv T_{2}^{2}\left(T_{2}+1\right)\left(\bmod T_{2}^{4}\right)$.

(f) Finally, by taking the third components at $v=2$ into account, we see that there is no relation containing $h_{1}^{\prime}$.

Recall that

$$
\begin{aligned}
\operatorname{dim} \operatorname{Sel}^{(2)}\left(\mathbb{Q}, J^{(p ; i, j)}\right)= & \operatorname{dim} \operatorname{val}^{-1}(G)+\operatorname{dim}\left(\operatorname{Im}\left(\delta_{2}\right) \times \operatorname{Im}\left(\delta_{p}\right)\right) \\
& -\operatorname{dim}\left(\left(\operatorname{Im}\left(\delta_{2}\right) \times \operatorname{Im}\left(\delta_{p}\right)\right)+\operatorname{res}_{S}\left(\operatorname{val}^{-1}(G)\right)\right)
\end{aligned}
$$

and

$$
J(\mathbb{Q}) / 2 J(\mathbb{Q}) \simeq \mathbb{F}_{2}^{\operatorname{rank}(J(\mathbb{Q}))} \oplus J(\mathbb{Q})[2] .
$$

Therefore, by Lemmas 2.14 to $2.16,2.19$ and 2.20 , we obtain

$$
\operatorname{rank}(J(\mathbb{Q})) \leq \operatorname{dim} \operatorname{Sel}^{(2)}(\mathbb{Q}, J)-\operatorname{dim} J(\mathbb{Q})[2]=7+6-11-2=0 .
$$

This completes the proof of Theorem $2.1(2)$.

2.3. Case $(i, j)=(0,2), p \equiv 3(\bmod 8)$

Suppose that $p \equiv 3(\bmod 8)$. Then, we have the following irreducible decompositions:

$$
\left\{\begin{array}{l}
L_{2}=\mathbb{Q}_{2}\left[T_{1}\right] /\left(T_{1}\right) \times \mathbb{Q}_{2}\left[T_{2}\right] /\left(T_{2}^{2}+p^{2}\right) \times \mathbb{Q}_{2}\left[T_{3}\right] /\left(T_{3}^{2}+2 p^{2}\right), \\
L_{p}=\mathbb{Q}_{p}\left[T_{1}\right] /\left(T_{1}\right) \times \mathbb{Q}_{p}\left[T_{2}\right] /\left(T_{2}^{2}+p^{2}\right) \times \mathbb{Q}_{p} \times \mathbb{Q}_{p} .
\end{array}\right.
$$

Here, we fix an element $\alpha \in \mathbb{Q}_{p}$ such that $\alpha^{2}=-2$, which is denoted by $\sqrt{-2}$. Moreover, we also fix an isomorphism $L_{p}^{(3)} \simeq \mathbb{Q}_{p} \times \mathbb{Q}_{p}$ which sends $T_{3}$ to $(p \sqrt{-2},-p \sqrt{-2})$. According to this irreducible decomposition, we denote each element in $L_{p}$ by the form $\left(\alpha_{1} ; \alpha_{2} ; \alpha_{3,1}, \alpha_{3,2}\right)$.

Lemma 2.21. The following two elements form an $\mathbb{F}_{2}$-basis of $J(\mathbb{Q})[2]$ and $J\left(\mathbb{Q}_{v}\right)[2]$ for $v=2, \infty$ :

$$
(0,0)-\infty, \quad \sum_{\substack{P \in C(\overline{\mathbb{Q}}) \\ x_{P}^{2}+p^{2}=0}} P-2 \infty .
$$

On the other hand, the following three elements form an $\mathbb{F}_{2}$-basis of $J\left(\mathbb{Q}_{p}\right)[2]$ :

$$
(0,0)-\infty, \quad(p \sqrt{-2}, 0)-\infty, \quad(-p \sqrt{-2}, 0)-\infty .
$$

In particular, we have the following table.

\begin{tabular}{|l||l|l|}
\hline$v$ & $\operatorname{dim} J\left(\mathbb{Q}_{v}\right)[2]$ & $\operatorname{dim} \operatorname{Im}\left(\delta_{v}\right)$ \\
\hline \hline 2 & 2 & 4 \\
\hline$p$ & 3 & 3 \\
\hline$\infty$ & 2 & 0 \\
\hline
\end{tabular}

Proof. The first and second statements follow from [12, Lemma 5.2]. Note that

- neither -1 nor -2 is square in $\mathbb{Q}_{v}$ for $v=2, \infty$.

- -2 is square in $\mathbb{Q}_{p}$ and -1 is not square in $\mathbb{Q}_{p}$.

The third statement follows from the following formula (cf. [4, p. 451, proof of Lemma $3])$.

$$
\operatorname{dim}_{\mathbb{F}_{2}} \operatorname{Im}\left(\delta_{v}\right)=\operatorname{dim}_{\mathbb{F}_{2}} J\left(\mathbb{Q}_{v}\right)[2] \begin{cases}+0 & (v \neq 2, \infty), \\ +2 & (v=2), \\ -2 & (v=\infty) .\end{cases}
$$


Lemma 2.22. The following three elements form a basis of $L_{p}^{\times} / L_{p}^{\times 2}$ form an $\mathbb{F}_{2}$-basis of $\operatorname{Im}\left(\delta_{p}\right)$ :

$\left(2 ; T_{2} ;-p \sqrt{-2}, p \sqrt{-2}\right), \quad\left(p \sqrt{-2} ; p \sqrt{-2}-T_{2} ; 1,-p \sqrt{-2}\right), \quad(-1 ; 1 ; p \sqrt{-2},-p \sqrt{-2})$.

In particular, the following three elements of $I_{p}(L) / I_{p}(L)^{2}$ form a basis of $G_{p}$ :

$$
((1) ;(p) ;(p),(p))_{p}, \quad((p) ;(p) ;(1),(p))_{p}, \quad((1) ;(1) ;(p),(p))_{p} .
$$

Proof. Lemma 2.4 implies that

$$
\begin{aligned}
\delta_{p}((0,0)-\infty) & =-T+\left(T^{2}+p^{2}\right)\left(T^{2}+2 p^{2}\right)=\left(2 ; T_{2} ;-p \sqrt{-2}, p \sqrt{-2}\right), \\
\delta_{p}((p \sqrt{-2}, 0)-\infty) & =-(T-p \sqrt{-2})+T(T+p \sqrt{-2})\left(T^{2}+p^{2}\right) \\
& =\left(p \sqrt{-2} ; p \sqrt{-2}-T_{2} ; 1,-p \sqrt{-2}\right), \\
\delta_{p}\left(\sum_{\substack{P \in C(\overline{\mathbb{Q}}) \\
x_{P}^{2}+2 p^{2}=0}} P-2 \infty\right) & =\left(T^{2}+2 p^{2}\right)-T\left(T^{2}+p^{2}\right)=(-1 ; 1 ; p \sqrt{-2},-p \sqrt{-2}) .
\end{aligned}
$$

Hence, the above three elements $\operatorname{lie} \operatorname{in} \operatorname{Im}\left(\delta_{p}\right)$. By taking their images in $G_{p}$ into account, we see that they are linearly independent. By Lemma 2.21, this completes the proof.

Lemma 2.23. The following four elements of $L_{2}^{\times} / L_{2}^{\times 2}$ form an $\mathbb{F}_{2}$-basis of $\operatorname{Im}\left(\delta_{2}\right)$ :

$$
\left(2 ; T_{2} ;-T_{3}\right), \quad\left(1 ; T_{2} ;-1\right), \quad\left(-2 ; 2+T_{2} ;-2-T_{3}\right), \quad\left(1 ; 1-4 T_{2} ; 1\right) .
$$

In particular, the following element of $I_{2}(L) / I_{2}(L)^{2}$ forms an $\mathbb{F}_{2}$-basis of $G_{2}$ :

$$
\left((2) ;(1) ;\left(T_{3}\right)\right)_{2} \text {. }
$$

Proof. First, we show that the above four elements actually lie in $\operatorname{Im}\left(\delta_{2}\right)$. Lemma 2.4 implies that

$$
\begin{array}{r}
\delta_{2}((0,0)-\infty)=-T+\left(T^{2}+p^{2}\right)\left(T^{2}+2 p^{2}\right)=\left(2 ; T_{2} ;-T_{3}\right), \\
\delta_{2}\left(\sum_{\substack{P \in C(\overline{\mathbb{Q}}) \\
x_{P}^{2}+p^{2}=0}} P-2 \infty\right)
\end{array}
$$

Hence, $\left(2 ; T_{2} ;-T_{3}\right),\left(1 ; T_{2} ;-1\right) \in \operatorname{Im}\left(\delta_{2}\right)$.

- Since $f(-2) \equiv 4(\bmod 32)$, there exists $Q \in C\left(\mathbb{Q}_{2}\right)$ such that $x_{Q}=-2$. In particular, $\left(-2 ; 2+T_{2} ;-2-T_{3}\right)=\delta_{2}(Q-\infty)$ lies in $\operatorname{Im}\left(\delta_{2}\right)$.

- Since $2^{10} f(1 / 4) \equiv 1(\bmod 8)$, there exists $R \in C\left(\mathbb{Q}_{2}\right)$ such that $x_{R}=1 / 4$. In particular, $\left(1 ; 1-4 T_{2} ; 1\right)=\delta_{2}(R-\infty)$ lies in $\operatorname{Im}\left(\delta_{2}\right)$.

Since $v_{2}(2)=v_{2}(-2)=1$, the first and third elements are non-trivial in $L^{\times} / L^{\times 2}$. Since -1 is non-trivial in $L_{2}^{(3) \times} / L_{2}^{(3) \times 2}$, the second element is non-trivial in $L^{\times} / L^{\times 2}$. In what follows, we show that $1-4 T_{2}$ is non-trivial in $L^{(2) \times} / L^{(2) \times 2}$ by contradiction.

Let $t$ be a uniformizer in $L_{2}^{(2)}$, and $b_{1}, b_{2} \in\{0,1\}$ such that $2 \equiv t^{2}+b_{1} t^{3}\left(\bmod t^{4}\right)$. Suppose that $1-4 T_{2} \equiv\left(1+a_{1} t+a_{2} t^{2}+\cdots\right)^{2}\left(\bmod t^{5}\right)$ with $a_{1}, a_{2} \in\{0,1\}$. Then, we have

$$
1+t^{4} \equiv 1+a_{1}^{2} t^{2}+a_{1} t^{3}+\left(a_{2}+a_{2}^{2}+a_{1} b_{1}\right) t^{4} \quad\left(\bmod t^{5}\right),
$$

which implies that $a_{1}=0$ and $a_{2}+a_{2}^{2}=1(\bmod 2)$, a contradiction. Thus, $\left(1 ; 1-4 T_{2} ; 1\right)$ is non-trivial in $L^{\times} / L^{\times 2}$. 
Finally, by taking the first and third components into account, the four elements in the statement are linearly independent.

Corollary 2.24. The following three elements of $L^{\times} / L^{\times 2}$ form an $\mathbb{F}_{2}$-basis of $\operatorname{Ker}(\mathrm{val})$ :

$$
(1 ; 1 ;-1), \quad\left(1 ; T_{2} / p ; 1\right), \quad(-1 ; 1 ; 1) .
$$

Proof. By the exactly same manner as in Corollaries 2.10 and 2.17, we obtain a short exact sequence

$$
1 \rightarrow \mathcal{O}_{L}^{\times} / \mathcal{O}_{L}^{\times 2} \rightarrow \operatorname{Ker}(\operatorname{val}) \rightarrow \operatorname{Cl}(L)[2] \rightarrow 0 .
$$

The lemma follows from the facts that $(1 ; 1 ;-1),\left(1 ; T_{2} / p ; 1\right)$ and $(-1 ; 1 ; 1)$ form an $\mathbb{F}_{2}$-basis of $\mathcal{O}_{L}^{\times} / \mathcal{O}_{L}^{\times 2}$, and the class number of $L^{(i)}$ is 1 for every $i=0,1,2$.

Lemma 2.25. The following four elements of $I(L) / I(L)^{2}$ form an $\mathbb{F}_{2}$-basis of $W=G$ :

$$
\begin{array}{rlr}
\left((2) ;(1) ;\left(T_{3}\right)\right)_{2} \times \mathbf{1}_{p}, & \mathbf{1}_{2} \times((1) ;(p) ;(p),(p))_{p}, \\
\mathbf{1}_{2} \times((p) ;(p) ;(1),(p))_{p}, & \mathbf{1}_{2} \times((1) ;(1) ;(p),(p))_{p} .
\end{array}
$$

Proof. Since the class number of $L^{(i)}$ is 1 for every $i=0,1$, we have $W=G$. Thus, the lemma follows from Lemmas 2.22 and 2.23 ,

Recall that there exists an exact sequence $1 \rightarrow \operatorname{Ker}(\mathrm{val}) \rightarrow \mathrm{val}^{-1}(G) \rightarrow W \rightarrow 1$. By Corollary 2.24 and Lemma 2.25 , we have the following lemma.

Lemma 2.26. Fix $a, b \in \mathbb{Z}$ such that $p=a^{2}+2 b^{2}$ in $L^{(3)}$. Then, the following seven elements of $L^{\times} / L^{\times 2}$ form an $\mathbb{F}_{2}$-basis of $\mathrm{val}^{-1}(G)$ :

$$
(1 ; 1 ;-1), \quad\left(1 ; T_{2} / p ; 1\right), \quad(-1 ; 1 ; 1), \quad\left(2 ; 1 ; T_{3}\right), \quad(1 ; p ; p), \quad\left(p ; p ; a+b \cdot T_{3} / p\right), \quad(1 ; 1 ; p) .
$$

Lemma 2.27. Set elements of $L_{2}^{\times} / L_{2}^{\times 2} \times L_{p}^{\times} / L_{p}^{\times 2}$ as follows:

$$
\begin{aligned}
& d_{1}:=\left(2 ; T_{2} ;-T_{3}\right)_{2} \times \mathbf{1}_{p}, \\
& d_{2}:=\left(1 ; T_{2} ;-1\right)_{2} \times \mathbf{1}_{p}, \\
& d_{3}:=\left(-2 ; 2+T_{2} ;-2-T_{3}\right)_{2} \times \mathbf{1}_{p}, \\
& d_{4}:=\left(1 ; 1-4 T_{2} ; 1\right)_{2} \times \mathbf{1}_{p}, \\
& d_{5}:=\mathbf{1}_{2} \times\left(2 ; T_{2} ;-p \sqrt{-2}, p \sqrt{-2}\right)_{p}, \\
& d_{6}:=\mathbf{1}_{2} \times\left(p \sqrt{-2} ; p \sqrt{-2}-T_{2} ; 1,-p \sqrt{-2}\right)_{p}, \\
& d_{7}:=\mathbf{1}_{2} \times(-1 ; 1 ; p \sqrt{-2},-p \sqrt{-2})_{p}, \\
& h_{1}:=\operatorname{res}_{S}(1 ; 1 ;-1)=(1 ; 1 ;-1)_{2} \times(1 ; 1 ;-1,-1)_{p}, \\
& h_{2}:=\operatorname{res}_{S}\left(1 ; T_{2} / p ; 1\right)=\left(1 ; T_{2} / p ; 1\right)_{2} \times\left(1 ; T_{2} / p ; 1,1\right)_{p}, \\
& h_{3}:=\operatorname{res}_{S}(-1 ; 1 ; 1)=(-1 ; 1 ; 1)_{2} \times(-1 ; 1 ; 1,1)_{p}, \\
& h_{4}:=\operatorname{res}_{S}\left(2 ; 1 ; T_{3}\right)=\left(2 ; 1 ; T_{3}\right)_{2} \times(2 ; 1 ; p \sqrt{-2},-p \sqrt{-2})_{p}, \\
& h_{5}:=\operatorname{res}_{S}(1 ; p ; p)=(1 ; p ; p)_{2} \times(1 ; p ; p, p)_{p}, \\
& h_{6}:=\operatorname{res}_{S}\left(p ; p ; a+b \cdot T_{3} / p\right)=\left(p ; p ; a+b \cdot T_{3} / p\right)_{2} \times(p ; p ; a+b \sqrt{-2}, a-b \sqrt{-2})_{p}, \\
& h_{7}:=\operatorname{res}_{S}(1 ; 1 ; p)=(1 ; 1 ; p)_{2} \times(1 ; 1 ; p, p)_{p} .
\end{aligned}
$$

(1) We have $d_{1} d_{2} d_{7} h_{4}=d_{2} d_{5} d_{7} h_{1} h_{2} h_{5} h_{7}=\mathbf{1}_{2} \times \mathbf{1}_{p}$.

(2) The twelve elements $d_{3}, \ldots, d_{7}, h_{1}, \ldots, h_{7}$ form an $\mathbb{F}_{2}$-basis of $\left(\operatorname{Im}\left(\delta_{2}\right) \times \operatorname{Im}\left(\delta_{p}\right)\right)+$ $\operatorname{res}_{S}\left(\operatorname{val}^{-1}(G)\right)$.

Proof. (1) We can check it by direct calculation. 
(2) By (1) and Lemmas 2.22, 2.23 and 2.26, $\left(\operatorname{Im}\left(\delta_{2}\right) \times \operatorname{Im}\left(\delta_{p}\right)\right)+\operatorname{res}_{S}\left(\operatorname{val}^{-1}(G)\right)$ is generated by $d_{3}, \ldots d_{7}, h_{1}, \ldots, h_{7}$. Set

$$
\begin{aligned}
& d_{3}=\left(-2 ; 2+T_{2} ;-2-T_{3}\right)_{2} \times \mathbf{1}_{p}, \\
& d_{4}=\left(1 ; 1-4 T_{2} ; 1\right)_{2} \times \mathbf{1}_{p}, \\
& d_{5}^{\prime}:=d_{5} d_{7} h_{1} h_{2} h_{5} h_{7}=\left(1 ; T_{2} ;-1\right)_{2} \times \mathbf{1}_{p}, \\
& d_{6}=\mathbf{1}_{2} \times\left(p \sqrt{-2} ; p \sqrt{-2}-T_{2} ; 1,-p \sqrt{-2}\right)_{p}, \\
& d_{7}^{\prime}:=d_{7} h_{4}=\left(2 ; 1 ; T_{3}\right)_{2} \times \mathbf{1}_{p}, \\
& h_{1}=(1 ; 1 ;-1)_{2} \times(1 ; 1 ;-1,-1)_{p}, \\
& h_{2}=\left(1 ; T_{2} / p ; 1\right)_{2} \times\left(1 ; T_{2} / p ; 1,1\right)_{p}, \\
& h_{3}^{\prime}:=h_{3} h_{4}=\left(-2 ; 1 ; T_{3}\right)_{2} \times(1 ; 1 ; p \sqrt{-2},-p \sqrt{-2})_{p}, \\
& h_{4}=\left(2 ; 1 ; T_{3}\right)_{2} \times(2 ; 1 ; p \sqrt{-2},-p \sqrt{-2})_{p}, \\
& h_{5}=(1 ; p ; p)_{2} \times(1 ; p ; p, p)_{p}, \\
& h_{6}=\left(p ; p ; a+b \cdot T_{3} / p\right)_{2} \times(p ; p ; a+b \sqrt{-2}, a-b \sqrt{-2})_{p}, \\
& h_{7}=(1 ; 1 ; p)_{2} \times(1 ; 1 ; p, p)_{p} .
\end{aligned}
$$

It is sufficient to prove that the above twelve elements are linearly independent.

(a) By taking the first components at $v=p$ into account, we see that there is no relation containing $d_{6}, h_{4}$ and $h_{6}$. 8

(b) By taking the second components at $v=p$ into account, we see that there is no relation containing $h_{2}$ and $h_{5}$.

(c) By taking the third and fourth components at $v=p$ into account, we see that there is no relation containing $h_{1}, h_{3}^{\prime}$ and $h_{7}$.

(d) By taking the first components at $v=2$ into account, we see that there is no relation containing $d_{3}$ and $d_{7}^{\prime}$.

(e) By taking the third components at $v=2$ into account, we see that there is no relation containing $d_{5}^{\prime}$.

(f) Finally, by taking the second components at $v=2$ into account, we see that there is no relation containing $d_{4}$.

Recall that

$$
\begin{aligned}
\operatorname{dim} \operatorname{Sel}^{(2)}\left(\mathbb{Q}, J^{(p ; i, j)}\right)= & \operatorname{dim} \operatorname{val}^{-1}(G)+\operatorname{dim}\left(\operatorname{Im}\left(\delta_{2}\right) \times \operatorname{Im}\left(\delta_{p}\right)\right) \\
& -\operatorname{dim}\left(\left(\operatorname{Im}\left(\delta_{2}\right) \times \operatorname{Im}\left(\delta_{p}\right)\right)+\operatorname{res}_{S}\left(\operatorname{val}^{-1}(G)\right)\right)
\end{aligned}
$$

and

$$
J(\mathbb{Q}) / 2 J(\mathbb{Q}) \simeq \mathbb{F}_{2}^{\operatorname{rank}(J(\mathbb{Q}))} \oplus J(\mathbb{Q})[2] .
$$

Therefore, by Lemmas 2.21 to 2.23 , 2.26 and 2.27, we obtain

$$
\operatorname{rank}(J(\mathbb{Q})) \leq \operatorname{dim} \operatorname{Sel}^{(2)}(\mathbb{Q}, J)-\operatorname{dim} J(\mathbb{Q})[2]=7+7-12-2=0 .
$$

This completes the proof of Theorem 2.1 (3).

\footnotetext{
${ }^{8}$ Note that the relations among our generators do NOT depend on $p$ whenever $p \equiv 3(\bmod 8)$ although the representation of $h_{6}$ depends on $a, b \in \mathbb{Z}$ such that $p=a^{2}+2 b^{2}$. It is similar in the cases $p \equiv-3,-1$ $(\bmod 8)($ cf. Lemma 2.34). In contrast, when $p \equiv 1(\bmod 8)$, the relations DO depend on $a, b, c, d \in \mathbb{Z}$ such that $p=a^{2}+2 b^{2}=c^{2}+d^{2}$.
} 
2.4. Case $(i, j)=(0,2), p \equiv-3(\bmod 8)$

Suppose that $p \equiv-3(\bmod 8)$. Then, we have the following irreducible decompositions:

$$
\left\{\begin{array}{l}
L_{2}=\mathbb{Q}_{2}\left[T_{1}\right] /\left(T_{1}\right) \times \mathbb{Q}_{2}\left[T_{2}\right] /\left(T_{2}^{2}+p^{2}\right) \times \mathbb{Q}_{2}\left[T_{3}\right] /\left(T_{3}^{2}+2 p^{2}\right), \\
L_{p}=\mathbb{Q}_{p}\left[T_{1}\right] /\left(T_{1}\right) \times \mathbb{Q}_{p} \times \mathbb{Q}_{p} \times \mathbb{Q}_{p}\left[T_{3}\right] /\left(T_{3}^{2}+2 p^{2}\right) .
\end{array}\right.
$$

Here, we fix an element $\beta \in \mathbb{Q}_{p}$ such that $\beta^{2}=-1$, which is denoted by $\sqrt{-1}$. Moreover, we also fix an isomorphism $L_{p}^{(2)} \simeq \mathbb{Q}_{p} \times \mathbb{Q}_{p}$ which sends $T_{2}$ to $(p \sqrt{-1},-p \sqrt{-1})$. According to this irreducible decomposition, we denote each element in $L_{p}$ by the form $\left(\beta_{1} ; \beta_{2,1}, \beta_{2,2} ; \beta_{3,2}\right)$.

Lemma 2.28. The following two elements form an $\mathbb{F}_{2}$-basis of $J(\mathbb{Q})[2]$ and $J\left(\mathbb{Q}_{v}\right)[2]$ for $v=2, \infty$ :

$$
(0,0)-\infty, \quad \sum_{\substack{P \in C(\overline{\mathbb{Q}}) \\ x_{P}^{2}+p^{2}=0}} P-2 \infty .
$$

On the other hand, the following three elements form an $\mathbb{F}_{2}$-basis of $J\left(\mathbb{Q}_{p}\right)[2]$ :

$$
(0,0)-\infty, \quad(p \sqrt{-1}, 0)-\infty, \quad(-p \sqrt{-1}, 0)-\infty .
$$

In particular, we have the following table.

\begin{tabular}{|l||l|l|}
\hline$v$ & $\operatorname{dim} J\left(\mathbb{Q}_{v}\right)[2]$ & $\operatorname{dim} \operatorname{Im}\left(\delta_{v}\right)$ \\
\hline \hline 2 & 2 & 4 \\
\hline$p$ & 3 & 3 \\
\hline$\infty$ & 2 & 0 \\
\hline
\end{tabular}

Proof. The first and second statements follow from [12, Lemma 5.2]. Note that

- neither -1 nor -2 is square in $\mathbb{Q}_{v}$ for $v=2, \infty$.

- -1 is square in $\mathbb{Q}_{p}$ and -2 is not square in $\mathbb{Q}_{p}$.

The third statement follows from the following formula (cf. 44, p. 451, proof of Lemma $3])$.

$$
\operatorname{dim}_{\mathbb{F}_{2}} \operatorname{Im}\left(\delta_{v}\right)=\operatorname{dim}_{\mathbb{F}_{2}} J\left(\mathbb{Q}_{v}\right)[2] \begin{cases}+0 & (v \neq 2, \infty) \\ +2 & (v=2) \\ -2 & (v=\infty)\end{cases}
$$

Lemma 2.29. The following three elements of $L_{p}^{\times} / L_{p}^{\times 2}$ form an $\mathbb{F}_{2}$-basis of $\operatorname{Im}\left(\delta_{p}\right)$.

$$
\left(2 ; p \sqrt{-1}, p \sqrt{-1} ; T_{3}\right), \quad\left(p \sqrt{-1} ; 1,2 p \sqrt{-1} ; p \sqrt{-1}-T_{3}\right), \quad(1 ; p \sqrt{-1}, p \sqrt{-1} ; 1) .
$$

In particular, the following three elements of $I_{p}(L) / I_{p}(L)^{2}$ form an $\mathbb{F}_{2}$ basis of $G_{p}$ :

$$
((1) ;(p),(p) ;(p))_{p}, \quad((p) ;(1),(p) ;(p))_{p}, \quad((1) ;(p),(p) ;(1))_{p} .
$$


Proof. Lemma 2.4 implies that

$$
\begin{aligned}
\delta_{p}((0,0)-\infty) & =-T+\left(T^{2}+p^{2}\right)\left(T^{2}+2 p^{2}\right)=\left(2 ; p \sqrt{-1}, p \sqrt{-1} ; T_{3}\right), \\
\delta_{p}((p \sqrt{-1}, 0)-\infty) & =(T-p \sqrt{-1})+T(T+p \sqrt{-1})\left(T^{2}+2 p^{2}\right) \\
& =\left(p \sqrt{-1} ; 2,2 p \sqrt{-1} ; p \sqrt{-1}-T_{3}\right) \\
\delta_{p}\left(\sum_{\substack{P \in C(\overline{\mathbb{Q}}) \\
x_{P}^{2}+p^{2}=0}} P-2 \infty\right) & =\left(T^{2}+p^{2}\right)-T\left(T^{2}+2 p^{2}\right)=(1 ; p \sqrt{-1}, p \sqrt{-1} ; 1) .
\end{aligned}
$$

Hence, the above three elements $\operatorname{lie}$ in $\operatorname{Im}\left(\delta_{p}\right)$. By taking their images in $G_{p}$ into account, we see that they are linearly independent. By Lemma 2.28, this completes the proof.

Lemma 2.30. The following four elements of $L_{2}^{\times} / L_{2}^{\times 2}$ form an $\mathbb{F}_{2}$-basis of $\operatorname{Im}\left(\delta_{2}\right)$ :

$$
\left(2 ; T_{2} ;-T_{3}\right), \quad\left(1 ; T_{2} ;-1\right), \quad\left(-2 ; 2+T_{2} ;-2-T_{3}\right), \quad\left(1 ; 1-4 T_{2} ; 1\right) .
$$

In particular, the following element of $I_{2}(L) / I_{2}(L)^{2}$ forms an $\mathbb{F}_{2}$-basis of $G_{2}$ :

$$
\left((2) ;(1) ;\left(T_{3}\right)\right)_{2} \text {. }
$$

Proof. See the proof of Lemma 2.23

Corollary 2.31. The following three elements of $L^{\times} / L^{\times 2}$ form an $\mathbb{F}_{2}$-basis of $\operatorname{Ker}(\mathrm{val})$ :

$$
(1 ; 1 ;-1), \quad\left(1 ; T_{2} / p ; 1\right), \quad(-1 ; 1 ; 1)
$$

Proof. See the proof of Corollary 2.24 .

Lemma 2.32. The following four elements of $I(L) / I(L)^{2}$ form an $\mathbb{F}_{2}$-basis of $W=G$ :

$$
\begin{array}{lll}
\left((2) ;(1) ;\left(T_{3}\right)\right)_{2} \times \mathbf{1}_{p}, & \mathbf{1}_{2} \times((1) ;(p),(p) ;(p))_{p}, & \\
& \mathbf{1}_{2} \times((p) ;(1),(p) ;(p))_{p}, & \mathbf{1}_{2} \times((1) ;(p),(p) ;(1))_{p} .
\end{array}
$$

Proof. See the proof of Lemma 2.25 .

Recall that there exists an exact sequence $1 \rightarrow \operatorname{Ker}(\operatorname{val}) \rightarrow \operatorname{val}^{-1}(G) \rightarrow W \rightarrow 1$. By Corollary 2.31 and Lemma 2.32, we have the following lemma.

Lemma 2.33. Fix $c, d \in \mathbb{Z}$ such that $p=c^{2}+d^{2}$ in $L^{(3)}$. Then, the following seven elements of $L^{\times} / L^{\times 2}$ form an $\mathbb{F}_{2}$-basis of $\mathrm{val}^{-1}(G)$ :

$$
(1 ; 1 ;-1), \quad\left(1 ; T_{2} / p ; 1\right), \quad(-1 ; 1 ; 1), \quad\left(2 ; 1 ; T_{3}\right), \quad(1 ; p ; p), \quad\left(p ; c+d \cdot T_{2} / p ; p\right), \quad(1 ; p ; 1) .
$$


Lemma 2.34. Set elements of $L_{2}^{\times} / L_{2}^{\times 2} \times L_{p}^{\times} / L_{p}^{\times 2}$ as follows:

$$
\begin{aligned}
& d_{1}:=\left(2 ; T_{2} ;-T_{3}\right)_{2} \times \mathbf{1}_{p}, \\
& d_{2}:=\left(1 ; T_{2} ;-1\right)_{2} \times \mathbf{1}_{p}, \\
& d_{3}:=\left(-2 ; 2+T_{2} ;-2-T_{3}\right)_{2} \times \mathbf{1}_{p}, \\
& d_{4}:=\left(1 ; 1-4 T_{2} ; 1\right)_{2} \times \mathbf{1}_{p}, \\
& d_{5}:=\mathbf{1}_{2} \times\left(2 ; p \sqrt{-1}, p \sqrt{-1} ; T_{3}\right)_{p}, \\
& d_{6}:=\mathbf{1}_{2} \times\left(p \sqrt{-1} ; 1,2 p \sqrt{-1} ; p \sqrt{-1}-T_{3}\right)_{p}, \\
& d_{7}:=\mathbf{1}_{2} \times(1 ; p \sqrt{-1}, p \sqrt{-1} ; 1)_{p}, \\
& h_{1}:=\operatorname{res}_{S}(1 ; 1 ;-1)=(1 ; 1 ;-1)_{2} \times \mathbf{1}_{p}, \\
& h_{2}:=\operatorname{res}_{S}\left(1 ; T_{2} / p ; 1\right)=\left(1 ; T_{2} / p ; 1\right)_{2} \times(1 ; \sqrt{-1}, \sqrt{-1} ; 1)_{p}, \\
& h_{3}:=\operatorname{res}_{S}(-1 ; 1 ; 1)=(-1 ; 1 ; 1)_{2} \times \mathbf{1}_{p}, \\
& h_{4}:=\operatorname{res}_{S}\left(2 ; 1 ; T_{3}\right)=\left(2 ; 1 ; T_{3}\right)_{2} \times\left(2 ; 1,1 ; T_{3}\right)_{p}, \\
& h_{5}:=\operatorname{res}_{S}(1 ; p ; p)=(1 ; p ; p)_{2} \times(1 ; p, p ; p)_{p}, \\
& h_{6}:=\operatorname{res}_{S}(p ; c+d \sqrt{-1} ; p)=\left(p ; c+d \cdot T_{2} / p ; T_{3}\right)_{2} \times\left(p ; c+d \sqrt{-1}, c-d \sqrt{-1} ; T_{3}\right)_{p}, \\
& h_{7}:=\operatorname{res}_{S}(1 ; p ; 1)=(1 ; p ; 1)_{2} \times(1 ; p, p ; 1)_{p} .
\end{aligned}
$$

(1) We have $d_{1} d_{2} d_{7} h_{4}=d_{2} d_{5} d_{7} h_{1} h_{2} h_{5} h_{7}=\mathbf{1}_{2} \times \mathbf{1}_{p}$.

(2) The twelve elements $d_{3}, \ldots, d_{7}, h_{1}, \ldots, h_{7}$ form an $\mathbb{F}_{2}$-basis of $\left(\operatorname{Im}\left(\delta_{2}\right) \times \operatorname{Im}\left(\delta_{p}\right)\right)+$ $\operatorname{res}_{S}\left(\operatorname{val}^{-1}(G)\right)$.

Proof. (1) We can check it by direct calculation.

(2) By (1) and Lemmas 2.29, 2.30 and 2.33. $\left(\operatorname{Im}\left(\delta_{2}\right) \times \operatorname{Im}\left(\delta_{p}\right)\right)+\operatorname{res}_{S}\left(\operatorname{val}^{-1}(G)\right)$ is generated by $d_{3}, \ldots d_{7}, h_{1}, \ldots, h_{7}$. Set

$$
\begin{aligned}
& d_{3}^{\prime}:=d_{3} d_{5} d_{7} h_{3} h_{4}=\left(1 ; 2+T_{2} ;\left(-2-T_{3}\right) T_{3}\right)_{2} \times \mathbf{1}_{p}, \\
& d_{4}=\left(1 ; 1-4 T_{2} ; 1\right)_{2} \times \mathbf{1}_{p}, \\
& d_{5}^{\prime}:=d_{5} d_{7} h_{4}=\left(2 ; 1 ; T_{3}\right)_{2} \times \mathbf{1}_{p}, \\
& d_{6}=\mathbf{1}_{2} \times\left(p \sqrt{-1} ; 1,2 p \sqrt{-1} ; p \sqrt{-1}-T_{3}\right)_{p}, \\
& d_{7}^{\prime}:=d_{7} h_{2} h_{7}=\left(1 ; T_{2} ; 1\right)_{2} \times \mathbf{1}_{p}, \\
& h_{1}=(1 ; 1 ;-1)_{2} \times \mathbf{1}_{p}, \\
& h_{2}=\left(1 ; T_{2} / p ; 1\right)_{2} \times(1 ; \sqrt{-1}, \sqrt{-1} ; 1)_{p}, \\
& h_{3}=(-1 ; 1 ; 1)_{2} \times \mathbf{1}_{p}, \\
& h_{4}=\left(2 ; 1 ; T_{3}\right)_{2} \times\left(2 ; 1,1 ; T_{3}\right)_{p}, \\
& h_{5}=(1 ; p ; p)_{2} \times(1 ; p, p ; p)_{p}, \\
& h_{6}=\left(p ; c+d \cdot T_{2} / p ; T_{3}\right)_{2} \times\left(p ; c+d \sqrt{-1}, c-d \sqrt{-1} ; T_{3}\right)_{p}, \\
& h_{7}=(1 ; p ; 1)_{2} \times(1 ; p, p ; 1)_{p} .
\end{aligned}
$$

It is sufficient to prove that the above twelve elements are linearly independent.

(a) By taking the first components at $v=p$ into account, we see that there is no relation containing $d_{6}, h_{4}$ and $h_{6}$.

(b) By taking the fourth components at $v=p$ into account, we see that there is no relation containing $h_{5}$.

(c) By taking the second and third components at $v=p$ into account, we see that there is no relation containing $h_{2}$ and $h_{7}$. 
(d) By taking the first components at $v=2$ into account, we see that there is no relation containing $d_{5}^{\prime}$, and $h_{3}$.

(e) By taking the third components at $v=2$ into account, we see that there is no relation containing $d_{3}^{\prime}$, and $h_{1}$.

(f) Finally, by taking the second components at $v=2$ into account, we see that there is no relation containing $d_{4}$ and $d_{7}^{\prime}$.

Therefore, by Lemmas 2.28 to $2.30,2.33$ and 2.34 , we obtain

$$
\operatorname{rank}(J(\mathbb{Q})) \leq \operatorname{dim} \operatorname{Sel}^{(2)}(\mathbb{Q}, J)-\operatorname{dim} J(\mathbb{Q})[2]=7+7-12-2=0 .
$$

This completes the proof of Theorem 2.1 (4).

\section{Application of the Lutz-Nagell type theorem for hyperelliptic curves}

Let $p$ be a prime number, $i, j \in \mathbb{Z}_{\geq 0}$, and $f(x)=x\left(x^{2}+2^{i} p^{j}\right)\left(x^{2}+2^{i+1} p^{j}\right)$. Let $C$ be a hyperelliptic curve defined by $y^{2}=f(x)$ and $J$ be its Jacobian variety.

By taking Theorem 2.1 into account, Theorem 1.1 is an immediate consequence of the following proposition.

Proposition 3.1. Let $P \in C(\mathbb{Q}) \backslash\{\infty\}$ such that $\phi(P) \in J(\mathbb{Q})_{\text {tors }}$.

(1) Suppose that $p \neq 3$. Then, $P=(0,0)$.

(2) Suppose that $p=3$ and $(i, j) \not \equiv(2,2),(3,2)(\bmod 4)$. Then, $P=(0,0)$.

This proposition follows from the following Lutz-Nagell type theorem.

Theorem 3.2 (cf. [5, Theorem 3]). Let $\phi: C \rightarrow J$ be the Abel-Jacobi map defined by $\phi(P)=[P-\infty]$. If $P=(a, b) \in C(\mathbb{Q}) \backslash\{\infty\}$ and $\phi(P) \in J(\mathbb{Q})_{\text {tors }}$, then $a, b \in \mathbb{Z}$ and either $b=0$ or $b^{2} \mid \operatorname{disc}(f)$.

Note that Grant [5] proved the above Lutz-Nagell type theorem in more general settings.

Proof of Proposition 3.1. (1) We prove this statement by contradiction. Suppose that $P=(a, b) \in C(\mathbb{Q}) \backslash\{\infty\}$ satisfies $\phi(P) \in J(\mathbb{Q})_{\text {tors }}$ and $b \neq 0$. Then, Theorem 3.2 implies that $a, b \in \mathbb{Z}$ and $b^{2} \mid \operatorname{disc}(f)$, hence $b= \pm 2^{k} p^{l}$ for some $k, l \in \mathbb{Z}_{\geq 0}$. In particular, every prime divisor of $f(a) \in \mathbb{Z}_{\geq 0}$ is 2 or $p$.

First, if $l=0$, then $f(a)$ is a power of 2 , which contradicts that $1<\left(a^{2}+\right.$ $\left.2^{i+1} p^{j}\right) /\left(a^{2}+2^{i} p^{j}\right)<2$. Thus, we may assume that $p$ is odd and $l>0$.

- Suppose that $j=0$. Then, $\operatorname{disc}(f)$ is divisible only by 2 , i.e. $l=0$, which is impossible.

Thus, $j$ must be positive, and there exists $a_{1} \in \mathbb{Z}$ such that $a=p a_{1}$, i.e.

$$
b^{2}=p^{3} a_{1}\left(p a_{1}^{2}+2^{i} p^{j-1}\right)\left(p a_{1}^{2}+2^{i+1} p^{j-1}\right) .
$$

- Suppose that $j=1$. Since $p$ is odd, neither $p a_{1}^{2}+2^{i}$ nor $p a_{1}^{2}+2^{i+1}$ is divisible by $p$. Thus, both $p a_{1}^{2}+2^{i}$ and $p a_{1}^{2}+2^{i+1}$ must be powers of 2 , which contradicts that $1<\left(p a_{1}^{2}+2^{i+1}\right) /\left(p a_{1}^{2}+2^{i}\right)<2$.

- Suppose that $j=2$. Then,

$$
b^{2}=p^{5} a_{1}\left(a_{1}^{2}+2^{i}\right)\left(a_{1}^{2}+2^{i+1}\right) .
$$

- Suppose that $i=0$. If $a_{1}$ is odd, then the right hand side is divisible by 2 exactly odd times, which is impossible. If $a_{1}$ is even, then $a_{1}^{2}+1>1$ and $\left(a_{1}^{2}+2\right) / 2>1$ must be coprime powers of $p$, which is impossible. 
- Suppose that $i=1$. If $a_{1}$ is odd, then $a_{1}^{2}+2>1$ and $a_{1}^{2}+4>1$ must be coprime powers of $p$, which is impossible. If $a_{1}$ is even, then there exists $a_{2} \in \mathbb{Z}$ such that $a_{1}=2 a_{2}$, i.e

$$
b^{2}=2^{4} p^{5} a_{2}\left(2 a_{2}^{2}+1\right)\left(a_{2}^{2}+1\right) .
$$

If $a_{2}$ is odd, then the right hand side is divisible by 2 exactly odd times, which is impossible. If $a_{2}$ is even, then $2 a_{2}^{2}+1>1$ and $a_{2}^{2}+1>1$ must be coprime powers of $p$, which is impossible.

- Suppose that $i=2$. If $a_{1}$ is odd, then $a_{1}^{2}+4>1$ and $a_{1}^{2}+8>1$ must be coprime powers of $p$, which is impossible. If $a_{1}$ is even, then there exists $a_{2} \in \mathbb{Z}$ such that $a_{1}=2 a_{2}$, i.e.

$$
b^{2}=2^{5} p^{5} a_{2}\left(a_{2}^{2}+1\right)\left(a_{2}^{2}+2\right) .
$$

If $a_{2}$ is odd, then $a_{2}$ and $a_{2}^{2}+2$ are coprime powers of $p$. Thus $a_{2}=1$, and the right hand side is divisible by $p \neq 3$ exactly odd times, which is impossible. If $a_{2}$ is even, then $a_{2}^{2}+1>1$ and $\left(a_{2}^{2}+2\right) / 2>1$ must be coprime powers of $p$, which is impossible.

- Suppose that $i=3$. If $a_{1}$ is odd, then $a_{1}^{2}+8>1$ and $a_{1}^{2}+16>1$ must be coprime powers of $p$, which is impossible. If $a_{1}$ is even, then there exists $a_{2} \in \mathbb{Z}$ such that $a_{1}=2 a_{2}$, i.e.

$$
b^{2}=2^{5} p^{5} a_{2}\left(a_{2}^{2}+2\right)\left(a_{2}^{2}+4\right) .
$$

If $a_{2}$ is odd, then $a_{2}^{2}+2>1$ must be $a_{2}^{2}+4>1$ are coprime powers of $p$, which is impossible. If $a_{2}$ is even, then there exists $a_{3} \in \mathbb{Z}$ such that $a_{2}=2 a_{3}$, i.e.

$$
b^{2}=2^{9} p^{5} a_{3}\left(2 a_{3}^{2}+1\right)\left(a_{3}^{2}+1\right) .
$$

If $a_{3}$ is odd, then $a_{3}$ and $2 a_{3}^{2}+1$ are coprime powers of $p$. Thus, $a_{3}=1$, and the right hand side is divisible by $p \neq 3$ exactly odd times, which is impossible. If $a_{3}$ is even, then $2 a_{3}^{2}+1>1$ and $a_{3}^{2}+1>1$ must be coprime powers of $p$, which is impossible.

- Suppose that $i \geq 4$. If $a_{1}$ is odd, then $a_{1}^{2}+2^{i}>1$ and $a_{1}^{2}+2^{i+1}>1$ must be coprime powers of $p$, which is impossible. If $a_{1}$ is even, then there exists $a_{2} \in \mathbb{Z}$ such that $a_{1}=2 a_{2}$, i.e.

$$
b^{2}=2^{5} p^{5} a_{2}\left(a_{2}^{2}+2^{i-2}\right)\left(a_{2}^{2}+2^{i-1}\right) .
$$

If $a_{2}$ is odd, then $a_{2}^{2}+2^{i-2}>1$ and $a_{2}^{2}+2^{i-1}>1$ must be coprime powers of $p$, which is a impossible. If $a_{2}$ is even, then there exist $a_{3}$, $b_{1} \in \mathbb{Z}$ such that $\left(a_{2}, b\right)=\left(2 a_{3}, 2^{5} b_{1}\right)$, i.e.

$$
b_{1}^{2}=p^{5} a_{3}\left(a_{3}^{2}+2^{i-4}\right)\left(a_{3}^{2}+2^{i-3}\right) .
$$

Therefore, by a simple induction on $i$, we obtain the claim.

- The case $j=3$ is similar to the case $j=1$.

- If $j \geq 4$, then there exist $a_{2}, b_{1} \in \mathbb{Z}$ such that $\left(a_{1}, b\right)=\left(p a_{2}, p^{5} b_{1}\right)$, i.e.

$$
b_{1}^{2}=a_{2}\left(a_{2}^{2}+2^{i} p^{j-4}\right)\left(a_{2}^{2}+2^{i+1} p^{j-4}\right) .
$$

Therefore, by a simple induction on $j$, we obtain the claim.

This completes the proof.

(2) The proof of (2) is exactly similar to (1). Note that, in the above proof, we used the condition $p \neq 3$ only in the cases $(i, j) \equiv(2,2),(3,2)(\bmod 4)$. 


\section{Concluding remarks}

If a hyperelliptic curve $C$ covers an elliptic curve $E$ over $\mathbb{Q}$, then we can determine $C(\mathbb{Q})$ by determining $E(\mathbb{Q})$. 9 However, our hyperelliptic curves in Theorem 1.1 do not cover elliptic curves over $\mathbb{Q}$. Indeed, the congruent zeta functions of $C^{(p ; i, j)}$ with $p \neq 11$ over $\mathbb{F}_{11}$ are

$$
Z\left(C^{(p ; i, j)} / \mathbb{F}_{11}, T\right)=\frac{1+14 T^{2}+121 T^{4}}{(1-T)(1-11 T)},
$$

whose numerators are irreducible in $\mathbb{Q}[T] .{ }^{10}$

On the other hand, it might be possible to prove Theorem 1.1 by determining the set of rational points of some elliptic curves over number fields covered by our hyperelliptic curves. There are two obstructions in this direction. First, it is a non-trivial problem to find such elliptic curves of Mordell-Weil rank 0. Secondly, since the unit group of number fields are infinite in general, the Lutz-Nagell theorem is not sufficient to determine the torsion points of elliptic curves over number fields.

\section{Appendix A.}

In this appendix, we prove the following proposition.

Proposition A.1. Let $i, j \in \mathbb{Z}_{\geq 0}$ and $C^{(i, j)}$ be a hyperelliptic curve over $\mathbb{Q}(t)$ defined by

$$
C^{(i, j)}: y^{2}=x\left(x^{2}+2^{i} t^{j}\right)\left(x^{2}+2^{i+1} t^{j}\right) .
$$

Then, $C^{(i, j)}(\mathbb{Q}(t))=\{(0,0), \infty\}$.

This is a consequence of the following "abc conjecture for polynomials".

Theorem A.2 (cf. [10, Theorem]). Let $k$ be a field of characteristic 0 , and $a, b$ and $c \in k[x]$ be coprime polynomials such that $a+b=c$. Suppose that $a, b$ and $c$ are not all constants. Then, $\operatorname{deg}(c)<n_{0}(a b c)$, where $n_{0}(a b c)$ is the number of distinct zeroes of abc.

Lemma A.3. There exist no $X, Y, Z \in \mathbb{Z}_{>0}$ such that

(1) $X^{4}+Y^{4}=Z^{2}$ or

(2) $X^{4}+4 Y^{4}=Z^{2}$.

Proof of Lemma A.3. (1) is a well-know theorem by Fermat.

We prove (2) by contradiction.

Suppose that there exist $X, Y, Z \in \mathbb{Z}_{>0}$ such that $X^{4}+4 Y^{4}=Z^{2}$. If $\operatorname{gcd}(X, Y)=$ $d \in \mathbb{Z}_{>0}$, then $Z$ is divisible by $d^{2}$. Thus, by replacing $X, Y, Z$ by $X / d, Y / d, Z / d^{2}$ if necessary, we may assume that $\operatorname{gcd}(X, Y, Z)=1$. Assume that $X$ is even. Since $\operatorname{gcd}\left(X^{2} / 2, Y^{2}, Z / 2\right)=1$, there exist $S, T \in \mathbb{Z}_{>0}$ such that $\operatorname{gcd}(S, T)=1$ and

$$
\left\{\begin{array}{l}
\frac{X^{2}}{2}=2 S T, \\
Y^{2}=S^{2}-T^{2}, \\
\frac{Z}{2}=S^{2}+T^{2} .
\end{array}\right.
$$

Since $\operatorname{gcd}(S, T)=1$, the first equality implies that there exist $U, V \in \mathbb{Z}_{>0}$ such that $S=U^{2}$ and $T=V^{2}$. The second equality implies that $Y^{2}=U^{4}-V^{4}$. Since $Y$ is odd, $U$

\footnotetext{
${ }^{9}$ For example, $y^{2}=\left(x^{2}+1\right)\left(x^{2}+1+p\right)\left(x^{2}+1-p\right)$ with a prime $p \equiv 3(\bmod 8)$ has no rational solution.

${ }^{10}$ Similarly, by calculating the congruent zeta functions over $\mathbb{F}_{13}$, we can check that $C^{(11 ; i, j)}$ do not cover elliptic curves over $\mathbb{Q}$.
} 
is odd and $V$ is even. Therefore, there exists $S^{\prime}, T^{\prime} \in \mathbb{Z}_{>0}$ such that $\operatorname{gcd}\left(S^{\prime}, T^{\prime}\right)=1$ and

$$
\left\{\begin{array}{l}
Y=S^{\prime 2}-T^{\prime 2}, \\
V^{2}=2 S^{\prime} T^{\prime}, \\
U^{2}=S^{\prime 2}+T^{\prime 2} .
\end{array}\right.
$$

The second equality implies that there exist $U^{\prime}, V^{\prime} \in \mathbb{Z}_{>0}$ such that $S^{\prime}=U^{\prime 2}$ and $T^{\prime}=V^{\prime 2}$. Therefore, $U^{2}=U^{\prime 4}+V^{\prime 4}$, which contradicts (1).

The case when $X$ is odd is similar.

Proof of Proposition A.1. Recall that $C^{(i, j)} \subset \mathbb{P}_{(1,3,1)}^{2}$ is defined by the following weighted homogeneous equation:

$$
Y^{2}=X Z\left(X^{2}+2^{i} t^{j} Z^{2}\right)\left(X^{2}+2^{i+1} t^{j} Z^{2}\right)
$$

We may assume that $X, Y, Z \in \mathbb{Q}[t]$. Put $t=s^{4}, U=s^{j} Y$ and $V=s^{2 j} Z$. Then, we obtain the curve $\tilde{C}^{(i, j)}$ defined by the following equation over $\mathbb{Q}(s)$ :

$$
U^{2}=X V\left(X^{2}+2^{i} V^{2}\right)\left(X^{2}+2^{i+1} V^{2}\right) .
$$

It is sufficient to prove that $\tilde{C}^{(i, j)}(\mathbb{Q}(s))=\{(0,0), \infty\}$. Let $P:=[X: U: V] \in C^{(i, j)}(\mathbb{Q}(s))$. We may assume that $X=X(s), U=U(s), V=V(s)$ are taken from $\mathbb{Z}[s]$ such that $\operatorname{gcd}(X, U, V)=1$. Then, there exist coprime polynomials $U_{1}, U_{2}, U_{3}, U_{4} \in \mathbb{Z}[s]$ such that

$$
\left\{\begin{array}{l}
X=U_{1}^{2} \\
V=U_{2}^{2} \\
X^{2}+2^{i} V^{2}=U_{3}^{2} \\
X^{2}+2^{i+1} V^{2}=U_{4}^{2}
\end{array}\right.
$$

Since $i$ or $i+1$ is even, there exists $U_{5} \in \mathbb{Z}[s]$ such that one of the following conditions holds.

$$
\left\{\begin{array}{lll}
U_{1}^{4}+U_{5}^{4}=U_{3}^{2} & (i \equiv 0 & (\bmod 4)) \\
U_{1}^{4}+4 U_{5}^{4}=U_{4}^{2} & (i \equiv 1 & (\bmod 4)) \\
U_{1}^{4}+4 U_{5}^{4}=U_{3}^{2} & (i \equiv 2 & (\bmod 4)) \\
U_{1}^{4}+U_{5}^{4}=U_{4}^{2} & (i \equiv 3 & (\bmod 4)) .
\end{array}\right.
$$

Suppose that $U_{1}^{4}+U_{5}^{4}=U_{3}^{2}$. Let $g:=\operatorname{gcd}\left(U_{1}, U_{3}, U_{5}\right) \in \mathbb{Z}_{>0}, U_{1}^{\prime}:=U_{1} / g, U_{3}^{\prime}:=U_{2} / g^{2}$, $U_{5}^{\prime}:=U_{5} / g$. If $U_{1}^{\prime}, U_{3}^{\prime}, U_{5}^{\prime}$ are not all constants, then Theorem A.2 implies that

$$
2 \operatorname{deg}\left(U_{3}^{\prime}\right)<n_{0}\left(U_{1}^{\prime} U_{3}^{\prime} U_{5}^{\prime}\right) \leq \operatorname{deg} U_{1}^{\prime}+\operatorname{deg} U_{3}^{\prime}+\operatorname{deg} U_{5}^{\prime} .
$$

Thus, we have

$$
\operatorname{deg}\left(U_{3}^{\prime 2}\right)<\operatorname{deg}\left(U_{1}^{\prime 2}\right)+\operatorname{deg}\left(U_{5}^{\prime 2}\right) \leq \operatorname{deg}\left(U_{1}^{\prime 4}+U_{5}^{\prime 4}\right),
$$

which is impossible. Therefore, $U_{1}^{\prime}, U_{3}^{\prime}, U_{5}^{\prime}$ are all integers such that $\operatorname{gcd}\left(U_{1}^{\prime}, U_{3}^{\prime}, U_{5}^{\prime}\right)=1$. Thus, by Lemma A.3 $(1),\left(U_{1}^{\prime}, U_{3}^{\prime}, U_{5}^{\prime}\right)=(0, \pm 1, \pm 1)$ or $( \pm 1, \pm 1,0)$, i.e. $P=[0: 0: 1]$ or $[1: 0: 0]$ as desired. The other cases are exactly similar. This completes the proof.

Acknowledgement. The authors thank their advisor Ken-ichi Bannai for reading the draft and giving helpful comments. The authors also thank him for warm and constant encouragement and professor Shuji Yamamoto for helpful comments.

\section{References}

[1] Wieb Bosma, John Cannon, and Catherine Playoust, The Magma algebra system. I. The user language, J. Symbolic Comput. 24 (1997), no. 3-4, 235-265, DOI 10.1006/jsco.1996.0125. Computational algebra and number theory (London, 1993). MR1484478 
[2] Robert F. Coleman, Effective Chabauty, Duke Math. J. 52 (1985), no. 3, 765-770, DOI 10.1215/S00127094-85-05240-8. MR808103

[3] David A. Cox, Primes of the form $x^{2}+n y^{2}$, 2nd ed., Pure and Applied Mathematics (Hoboken), John Wiley \& Sons, Inc., Hoboken, NJ, 2013. Fermat, class field theory, and complex multiplication. MR3236783

[4] E. V. Flynn, Bjorn Poonen, and Edward F. Schaefer, Cycles of quadratic polynomials and rational points on a genus-2 curve, Duke Math. J. 90 (1997), no. 3, 435-463, DOI 10.1215/S0012-7094-9709011-6. MR1480542

[5] David Grant, On an analogue of the Lutz-Nagell theorem for hyperelliptic curves, J. Number Theory 133 (2013), no. 3, 963-969, DOI 10.1016/j.jnt.2012.02.023. MR2997779

[6] Richard K. Guy, Unsolved problems in number theory, 3rd ed., Problem Books in Mathematics, Springer-Verlag, New York, 2004. MR2076335

[7] William McCallum and Bjorn Poonen, The method of Chabauty and Coleman, Explicit methods in number theory, Panor. Synthèses, vol. 36, Soc. Math. France, Paris, 2012, pp. 99-117 (English, with English and French summaries). MR3098132

[8] Patrick Morton, On Rédei's theory of the Pell equation, J. Reine Angew. Math. 307/308 (1979), 373-398, DOI 10.1515/crll.1979.307-308.373. MR534233

[9] Edward F. Schaefer, 2-descent on the Jacobians of hyperelliptic curves, J. Number Theory 51 (1995), no. 2, 219-232, DOI 10.1006/jnth.1995.1044. MR1326746

[10] Noah Snyder, An alternate proof of Mason's theorem, Elem. Math. 55 (2000), no. 3, 93-94, DOI $10.1007 / \mathrm{s} 000170050074$. MR1781918

[11] Michael Stoll, Implementing 2-descent for Jacobians of hyperelliptic curves, Acta Arith. 98 (2001), no. 3, 245-277, DOI 10.4064/aa98-3-4. MR1829626

[12] _ Arithmetic of hyperelliptic curves (unpublished lecture note), available from http://www.mathe2.uni-bayreuth.de/stoll/teaching/ArithHypKurven-SS2014/SkriptArithHypCurves-pub-screen.pdf.

(Yoshinosuke Hirakawa) Department of Science and Technology, Keio University, 14-1, Hiyoshi 3-CHOMe, KOUHOKU-KU, YOKOHAMA-Shi, KANAGAWA-Ken, JAPAN

E-mail address: hirakawa@keio.jp

(Hideki Matsumura) Department of Science and Technology, Keio University, 14-1, Hiyoshi 3-ChOme, Kouhoku-Ku, Yokohama-shi, Kanagawa-ken, Japan

E-mail address: hidekimatsumura@keio.jp 